\title{
Genetic and Epigenetic Regulation of Skeletal Muscle Ribosome Biogenesis with Exercise
}

Authors:

Affiliations:

Running Title:

\author{
Vandré C. Figueiredo $\mathrm{PhD}^{1,2}$, Yuan Wen $\mathrm{MD}, \mathrm{PhD}^{2,3}$, \\ Björn Alkner MD, $\mathrm{PhD}^{4}$, Rodrigo Fernandez-Gonzalo $\mathrm{PhD}^{5}$, \\ Jessica Norrbom $\mathrm{PhD}^{6}$, Ivan J. Vechetti Jr $\mathrm{PhD}^{2,7}$, \\ Taylor Valentino $\mathrm{MSc}^{2,3}$, C. Brooks Mobley $\mathrm{PhD}^{2,3}$, Gabriel E. Zentner \\ Charlotte A. Peterson PhD ${ }^{1,2,3}$, John J. McCarthy PhD ${ }^{2,3}$, \\ Kevin A. Murach $\mathrm{PhD}^{1,2 *}$, Ferdinand von Walden $\mathrm{MD}, \mathrm{PhD}^{2,3,9 *}$
}

1 College of Health Sciences, University of Kentucky, Lexington, KY, USA.

2 The Center for Muscle Biology, University of Kentucky, Lexington, KY, USA.

3 Department of Physiology, University of Kentucky, Lexington, KY, USA.

$4 \quad$ Department of Orthopaedics, Eksjö, Region Jönköping County and Department of Biomedical and Clinical Sciences, Linköping University, Linköping, Sweden.

5 Division of Clinical Physiology, Department of Laboratory Medicine, Karolinska Institutet, and Unit of Clinical Physiology, Karolinska University Hospital, Stockholm, Sweden.

6 Department of Physiology and Pharmacology, Karolinska Institutet, Stockholm, Sweden.

7 Department of Nutrition and Health Sciences, University of Nebraska, Lincoln, NE, USA

8 Department of Biology, Indiana University, Bloomington, IN, USA

9 Division of Pediatric Neurology, Department of Women's and Children's Health, Karolinska Institutet, Stockholm, Sweden.

rDNA Regulation with Exercise

\section{Senior/Corresponding Authors}

*Senior/Corresponding author:

Ferdinand von Walden, MD, PhD.

Dept. of Women's and Children's health, Karolinska Institute

ALB Q2:07, Karolinska University Hospital

17176 Stockholm

SWEDEN

Tel: +46-70-783-14-89

Email: Ferdinand.von.walden@ki.se

${ }^{*}$ Co-Senior/Co-Corresponding Author

Kevin A. Murach, PhD

Department of Physical Therapy

900 South Limestone CTW 445

Lexington, Kentucky 40536

UNITED STATES

Email: kmu236@g.uky.edu 


\section{ABSTRACT}

53 Ribosomes are the macromolecular engines of protein synthesis. Skeletal muscle ribosome

54 biogenesis is stimulated by exercise, but the contribution of ribosomal DNA (rDNA) copy number

55 and methylation to exercise-induced rDNA transcription is unclear. To investigate the genetic and

56 epigenetic regulation of ribosome biogenesis with exercise, a time course of skeletal muscle

57 biopsies was obtained from 30 participants (18 men and 12 women; $31 \pm 8 \mathrm{yrs}, 25 \pm 4 \mathrm{~kg} / \mathrm{m}^{2}$ ) at

58 rest and $30 \mathrm{~min}, 3 \mathrm{~h}, 8 \mathrm{~h}$, and $24 \mathrm{~h}$ after acute endurance $\left(\mathrm{n}=10,45 \mathrm{~min}\right.$ cycling, $\left.70 \% \mathrm{VO}_{2} \mathrm{max}\right)$ or

59 resistance exercise ( $n=10,4 \times 7 \times 2$ exercises); 10 control participants underwent biopsies

60 without exercise. rDNA transcription and dosage were assessed using qPCR and whole genome

61 sequencing. rDNA promoter methylation was investigated using massARRAY EpiTYPER, and

62 global rDNA CpG methylation was assessed using reduced-representation bisulfite sequencing.

63 Ribosome biogenesis and MYC transcription were associated with resistance but not endurance

64 exercise, indicating preferential up-regulation during hypertrophic processes. With resistance

65 exercise, ribosome biogenesis was associated with rDNA gene dosage as well as epigenetic

66 changes in enhancer and non-canonical MYC-associated areas in rDNA, but not the promoter. A

67 mouse model of in vivo metabolic RNA labeling and genetic myonuclear fluorescent labeling

68 validated the effects of an acute hypertrophic stimulus on ribosome biogenesis and Myc

69 transcription, and corroborated rDNA enhancer and Myc-associated methylation alterations

70 specifically in myonuclei. This study provides the first information on skeletal muscle genetic and

71 rDNA gene-wide epigenetic regulation of ribosome biogenesis in response to exercise, revealing

72 novel roles for rDNA dosage and CpG methylation. 


\section{Acute}

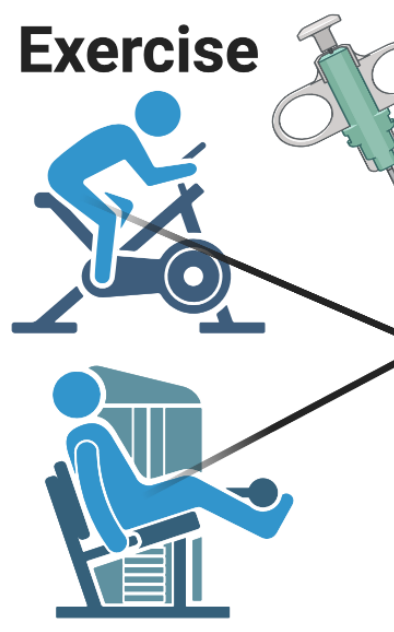

$\mathrm{n}=20$
VL Biopsy (Bx)

Pre, 30 min,

Whole Genome Sequencing, mRNA, and Protein

\section{Endurance (EE)}

$\leftrightarrow$ Ribosome Biogenesis

(RiboBio)

3h, 8h, 24h post biopsies (Bx)

Labeled RNA

Mouse model for

in vivo metabolic RNA labeling and fluorescent myonuclear labeling

+ acute hypertrophic stimulus

$\checkmark$ Myonuclear sorting + nucleus type-specific RRBS

\section{$\uparrow$ nascent rRNA and $M y c$ mRNA} $\uparrow M Y C$ mRNA

Differential methylation in enhancer, intergenic spacer, and MYC-associated areas 


\section{INTRODUCTION}

90 Ribosomes are the molecular factories responsible for protein synthesis, which is a key process

91 in the long-term adaptive response to exercise in skeletal muscle (Figueiredo, 2019a; McCarthy

92 \& Murach, 2019). Ribosome biogenesis is stimulated by muscle loading (Figueiredo \& McCarthy,

93 2019b; Kim et al., 2019; von Walden, 2019b), and the magnitude of de novo synthesis of

94 ribosomes is proportional to the amount of load-induced adult muscle hypertrophy in both

95 rodents (Nakada et al., 2016) and humans (Figueiredo et al., 2015; Stec et al., 2016;

96 Hammarstrom et al., 2020). Mechanical loading rapidly induces RNA Polymerase I (Pol I)

97 transcription of ribosomal DNA (rDNA), as assessed by $45 S$ pre-rRNA transcription levels and

98 accumulation of rRNA (von Walden et al., 2012b; Kirby et al., 2016; Figueiredo et al., 2019b).

99 The production of the long 45S pre-rRNA transcript, which is processed into the mature 18S,

$1005.8 \mathrm{~S}$ and 28S rRNAs, is believed to be the rate limiting step of ribosome biogenesis (Moss

101 2004). Contrary to protein-coding genes that commonly occur in the human genome as two

102 copies, rDNA genes number in the hundreds and vary widely across individuals (Gibbons et al.,

103 2015; Malinovskaya et al., 2018; Parks et al., 2018). While each rDNA locus can participate in

104 the formation of the nucleolus, the subnuclear compartment where Pol I transcribes 45S pre-

105 rRNA, but nothing is known about how rDNA copy number (dosage) affects ribosome biogenesis

106 in muscle. Furthermore, while ribosome biogenesis is implicated in the muscle hypertrophic

107 process, little is known about its contribution to the endurance exercise response.

108 We recently showed that an acute hypertrophic stimulus results in widespread CpG

109 hypomethylation in promoter sites of genes related to growth (i.e. mTOR pathway and Myc)

110 specifically within muscle fiber nuclei (myonuclei) (Von Walden et al., 2020a). These data concur

111 with earlier findings showing epigenetic and signaling-related regulation of rDNA promoter

112 regions in response to hypertrophic stimuli in vivo and in vitro in mice (von Walden et al., 2012;

113 von Walden et al., 2016). Thus, early dynamic epigenetic events associate with robust

114 transcriptional responses required for successful adaptation to exercise (Jozsi et al., 2000;

115 Pilegaard et al., 2000). rDNA is highly regulated at the epigenetic level in general (Grummt,

116 2007; Murayama et al., 2008), but it is currently unknown whether rDNA promoter

117 hypomethylation contributes to rRNA accumulation in response to exercise. Furthermore,

118 epigenetic patterning and regulation of rDNA transcription is unique in part due to its tandem

119 repeat organization and large intergenic spacer (IGS) (Baldridge et al., 1992; Mougey et al.,

120 1996; Zentner et al., 2011a; Audas et al., 2012; Shiue et al., 2014), but there is no information on

121 whether methylation of alternative regulatory sites in the rDNA repeat, such as enhancer regions 
122 and non-canonical transcription factor binding areas, are affected by mechanical loading and

123 associate with ribosome biogenesis in muscle.

124 In the current investigation, we present the first time-course of ribosome biogenesis and rRNA

125 transcription regulatory factor responses to acute endurance and resistance exercise (EE and

126 RE, respectively) in human skeletal muscle, and comprehensively evaluate how rDNA gene

127 dosage and methylation relates to rDNA transcription. To accomplish this, we employed whole-

128 genome sequencing, targeted mass spectrometry-based rDNA promoter methylation analysis,

129 reduced-representation bisulfite sequencing (RRBS), and mRNA- and protein-level measures.

130 We complemented the human analyses with an analogous murine model of acute muscle

131 loading, in vivo metabolic RNA labeling, and myonuclear-specific RRBS. With these parallel

132 approaches, we reveal novel information on genetic and epigenetic transcriptional control of the

133 translational apparatus following exercise. Our findings may have implications for individual

134 heterogeneity in exercise responsiveness to training (Ahtiainen et al., 2016; Sparks, 2017; Lavin

135 et al., 2019), as well as the epigenetic mechanisms of potentiated exercise adaptability related to

136 long-term cellular "muscle memory" (Murach et al., 2019; Murach et al., 2020; Snijders et al.,

137 2020). 


\section{METHODS}

\section{Research participants}

140 Thirty healthy male and female participants (18 males and 12 females) were recruited and

141 randomized to either a control $(C O N, n=10)$, an endurance exercise $(E E, n=10)$ or a resistance

142 exercise group (RE, $n=10)$. All groups included six males and four females. Participant

143 characteristics are presented in Table 1. All participants were recreationally active, i.e. involved

144 in EE one to three times per week and/or RE one to two times per week. Inclusion criterion was

145 18-50 years of age, and exclusion criteria were cardiovascular disease, neuromuscular disease,

146 or severe knee problems. The study protocol was approved by the Regional Ethical Review

147 board in Linköping and conformed to the Declaration of Helsinki. After receiving written and oral

148 information about the study, the participants gave their informed consent to participate.

\section{Human study design}

150 At least five days prior to the intervention, participants were familiarized with the experimental 151 set-up. All subjects performed a submaximal test on a cycle egometer (Monark $828 \mathrm{E}$, Monark

152 Exercise $\mathrm{AB}$, Vansbro, Sweden), to estimate $\mathrm{VO}_{2} \max$ (Ekblom-Bak et al., 2014; Bjorkman et al.,

153 2016) and seven repetition maximum (7RM) for knee extension and leg press was titrated.

154 These data were used to determine the load for the acute exercise bouts and to characterize the 155 three groups with respect to physical status. Subjects were instructed not to perform any

156 strenuous resistance for the legs three days prior to the intervention and no training the day prior.

157 A liquid formula (1.05 g carbohydrates/kg body weight (bw), $0.28 \mathrm{~g}$ protein/kg bw, and $0.25 \mathrm{~g}$

$158 \mathrm{fat} / \mathrm{kg} \mathrm{bw}$ ) was provided as breakfast $1 \mathrm{~h}$ prior to collection of the pre-exercise sample and as

159 lunch ( $2.10 \mathrm{~g}$ carbohydrates $/ \mathrm{kg}$ body weight (bw), $0.56 \mathrm{~g}$ protein $/ \mathrm{kg} \mathrm{bw}$, and $0.50 \mathrm{~g} \mathrm{fat} / \mathrm{kg} \mathrm{bw}$ )

160 immediately after the $3 \mathrm{~h}$ biopsy and at breakfast on day $2,2 \mathrm{~h}$ before the final biopsy. The liquid

161 formulas contained $5.6 \mathrm{~g}$ protein, $21 \mathrm{~g}$ carbohydrates, and $5.0 \mathrm{~g}$ fat per $100 \mathrm{ml}$ (Resource

162 komplett Näring, Nestlé Health Science, Stockholm, Sweden). Subjects were instructed to eat a

163 standard dinner (plate model) the evening before the experiment and in the evening on the day

164 of the experiment (Camelon et al., 1998). Skeletal muscle biopsies were collected before the

165 intervention and at $30 \mathrm{~min}, 3 \mathrm{~h}, 8 \mathrm{~h}$ and $24 \mathrm{~h}$ post exercise (Fig 1A). Eight subjects had 1-2 less

166 biopsies taken due to various reasons (e.g. failed biopsy, adverse event) which affected the

167 sample size for some analyses, and in some cases, there was only enough material for a specific

168 analysis. Muscle biopsies were obtained from the vastus lateralis muscle percutaneously after

169 injection of local anesthetic (carbocain $10 \mathrm{mg} / \mathrm{ml}$ ), by using the Bergström biopsy needle with a

170 diameter of $5 \mathrm{~mm}$ (Stille AB, Torshälla, Sweden). Skeletal muscle tissue was blotted for excess 
171 blood, cleaned of non-skeletal muscle tissue and snap-frozen in liquid nitrogen. All samples were

172 stored at $-80^{\circ} \mathrm{C}$ until further analysis.

\section{Human exercise protocols}

174 RE consisted of two lower limb exercises; leg press (Nordic Gym AB, Bollnäs, Sweden) and 175 knee extension (Nordic Gym AB). After a short warm-up on submaximal loads, the participants

176 performed 4 sets per exercise at 7 repetition maximum load with 2 min rest between sets and 5

177 min between exercises. EE consisted of 45 min cycling (Monark $828 \mathrm{E}$, Monark Exercise AB,

178 Vansbro, Sweden) at $70 \%$ of estimated $\mathrm{VO}_{2 \max }$. Heart rate was monitored continuously (Garmin

179 Edge 25, Garmin, United States) and participants were asked to rate their level of perceived

180 exertion every 5 min using the Borg RPE scale (Borg, 1970).

\section{$181 \quad$ Western blotting}

182 Protein was extracted from the organic phase of TRI Reagent following RNA extraction using the 183 optimized protocol (Wen et al., 2020). Briefly, following the final step of ethanol addition, samples 184 were centrifuged and the resulting protein pellet solubilized using SDS-urea buffer (100 mM Tris, $185 \mathrm{pH} 6.8,12 \%$ glycerol, 4\% SDS, 0.008\% bromophenol blue, $2 \% \beta$-mercaptoethanol, $5 \mathrm{M}$ urea)

186 supplemented with Halt ${ }^{\mathrm{TM}}$ Protease (ThermoFisher \#78438) and Phosphatase (\#78426) Inhibitor

187 Cocktails. RC DC TM Protein Assay (Bio-Rad, Hercules, CA) was used to determine protein

188 concentration. Twenty micrograms of protein per sample was loaded on a gradient gel

189 (Criterion ${ }^{\mathrm{TM}}$ Precast Gels, Bio-Rad) and electrophoretically transferred to a PVDF membrane

190 (Bio-Rad). Pool control samples were loaded on all gels. Membranes were blocked in 5\% bovine

191 serum albumin (BSA, \#A-420-1, Gold Biotechnology, St. Louis, MO), for phospho-specific

192 antibodies, or 5\% Non-Fat dry milk (\#170-6404, Bio-Rad), for pan-antibodies, in Tris-buffered

193 saline (TBS) with $0.1 \%$ Tween 20 (TBS-T) for two hours at room temperature. Following

194 blocking, membranes were incubated overnight at $4^{\circ} \mathrm{C}$ with a primary antibody (all Cell Signaling

195 Technology, Inc., Danvers, MA) in blocking solution. Antibodies used were: phospho-p70S6

196 Kinase (Thr389, 108D2, \#9234, dilution 1:2000), pan-p70S6 Kinase (\#9202, 1:2000), phospho-

197 S6 Ribosomal Protein (Ser240/244, D68F8, \#5364, dilution 1:2,000), pan-S6 Ribosomal Protein

198 (5G10, \#2217, dilution 1:3000), phospho-AMPKa (Thr172, 40H9, \#2535, dilution 1:1000), pan-

199 AMPKa (\#2532, dilution 1:2000). The next day, membranes were incubated with a goat anti-

200 rabbit (\#G-21234, Thermo Fisher Scientific) secondary antibody (dilution 1:10,000 in blocking

201 solution) for 1 hour at room temperature. Membranes were incubated with enhanced

202 chemiluminescence (ECL) reagent (Clarity Western ECL substrate, \#170-5060, Bio-Rad) before 
203 exposure to a ChemiDoc ${ }^{\mathrm{TM}}$ MP Imaging System (Bio-Rad). Bands were quantified using ImageJ

204 software (NIH, Bethesda, MD). Coomassie blue staining was utilized to confirm equal loading.

205 RNA extraction, cDNA synthesis and gene expression analysis

206 Approximately 25 mg of muscle tissue was used to extract RNA using TRI Reagent ${ }^{\circledR}$ (Sigma-

207 Aldrich, St. Louis, MO). Tissue was homogenized using beads and the Bullet Blender $囚$ Tissue

208 Homogenizer (Next Advance, Troy, NY). Following homogenization, RNA was isolated via phase

209 separation by addition of bromochloropropane (BCP) and centrifugation. The supernatant was

210 then transferred to a new tube and further processed on columns using the Direct-zol ${ }^{\mathrm{TM}} \mathrm{Kit}$

211 (Zymo Research, Irvine, CA, USA). RNA was treated in-column with DNAse and eluted in

212 nuclease-free water before being stored at $-80^{\circ} \mathrm{C}$. For quantitative reverse transcription PCR

213 (qRT-PCR) 750 ng of total RNA was reverse transcribed using SuperScript ${ }^{\mathrm{TM}}$ IV VILO ${ }^{\mathrm{TM}}$ Master

214 Mix (Invitrogen Carlsbad, CA). SsoAdvanced Universal SYBR Green Supermix (Bio-Rad,

215 Hercules, CA) was used for quantitative reverse transcription PCR (qRT-PCR), on the CFX384

216 Thermocycler (Bio-Rad). PCR data was normalized by the geometric mean of three stable

217 reference genes (EMC7, VCP, C1ORF43). Primer sequences are available upon request.

218 Melting curves were performed for every primer pair to confirm a single-product amplification.

219 qRT-PCR data were analyzed using the $2^{-\Delta \Delta}$ CT method.

\section{Targeted human rDNA promoter methylation analysis}

221 Genomic DNA was isolated from muscle samples using the QIAamp DNA Mini kit (Qiagen,

222 Hilden, Germany) according to the manufacturer's protocol. In brief, $25 \mathrm{mg}$ frozen muscle

223 samples were incubated and homogenized by enzyme digestion and mechanical disruption.

224 After tissue had been completely dissolved, the mixture was added to mini spin-columns and a

225 series of wash steps was performed. Each sample was diluted in $70 \mu$ of distilled water.

226 Immediately after DNA had been extracted, quantity and quality were determined in a

227 NanoPhotometer NP80 (Implen, München, Germany).

228 Quantitative methylation analysis was performed using the EpiTYPER methodology (Ehrich et al.

229 2005) and the MassARRAY® system (Agena Biosciences, San Diego, CA, USA) according to

230 manufacturer's recommendations and protocols, as previously described by our laboratory (von

231 Walden et al. 2020b). In this method, a targeted amplification of bisulfite converted DNA is

232 followed by in vitro transcription, RNase cleavage and subsequent fragment mass analysis by

233 Matrix-Assisted Laser Desorption/lonization Time of Flight Mass Spectrometry (MALDI-TOF MS)

234 to quantify CpG sites. PCR primers were adapted from D'Aquilla et al. 2017 (D'Aquila et al., 
235 2017). EpiTect methylated and non-methylated bisulfite-treated control DNA (Qiagen) was used

236 to evaluate the quantitative recapture of methylation ratios of the amplicons. The amplicon used

237 in this study met the quality criteria of methylated and non-methylated data points measured at $>$

$23879 \%$ and $<5 \%$ methylation ratios, respectively, as well as standard deviation percentages $<5 \%$.

239 Samples were run in duplicate and standard deviation percentages $>20 \%$ were removed from

240 the study (six out of 30 participants). The remaining data points (from $n=24$ participants)

241 correlated with $R^{2}$ 0.72. Bisulfite conversion efficiency was evaluated by analyzing one non-CpG

242 C's in a subset of the study samples. All data were checked by manually and visually inspecting

243 the mass spectra.

\section{Estimation of rDNA copy number via quantitative PCR (qPCR)}

245 Relative ribosomal DNA copy number was estimated by qPCR (rDNA dosage). Genomic (g)DNA

246 was extracted from muscle biopsies (from $n=27$ participants) and isolated using the Monarch kit

247 for DNA isolation (New England Biolabs, Ipswich, MA) according to the manufacturer's

248 instructions. Proteinase K digestion was performed overnight, and all samples were RNAse

249 treated before being purified on column. gDNA was eluted in nuclease-free $\mathrm{H}_{2} \mathrm{O}$ and diluted to

250 same concentration. $2.5 \mathrm{ng}$ of DNA was loaded per well in triplicate. qPCR was run using Fast

251 SYBR Green Master Mix (Applied Biosystems ${ }^{\mathrm{TM}}$, Foster City, CA) in a QuantStudio 3 Real-Time

252 PCR Systems (Thermo Fisher Scientific, Waltham, MA). The sequence of the primers (18S, 5.8S

253 and 28S, 5S and TP53 as reference gene), utilized in this study to assess rDNA dosage, are

254 from (Gibbons et al., 2015): TP53 F 5'TGTCCTTCCTGGAGCGATCT3' and R

255 5'CAAACCCCTGGTTTAGCACTTC3'; 5S rDNA F 5'TCGTCTGATCTCGGAAGCTAA3' and R

256 5'AAGCCTACAGCACCCGGTAT3'; 5.8S rDNA F 5'CGACTCTTAGCGGTGGATCA3' and R

257 5'GATCAATGTGTCCTGCAATTC3'; 18S rDNA F 5'GACTCAACACGGGAAACCTC3' and R

258 5'AGACAAATCGCTCCACCAAC3'; 28S rDNA F 5'GCGGGTGGTAAACTCCATCT3' and R

259 5'CACGCCCTCTTGAACTCTCT3'. Data were normalized to TP53 and expressed in arbitrary

260 units (AU). Data were not compared to a standard curve with known rDNA quantity and is

261 therefore referred to as relative rDNA dosage. Sample number is smaller $(n=7)$ when comparing

262 rDNA gene dosage to rDNA transcription at 24h post exercise due to technical reasons outlined

263 above, and DNA for one sample not being suitable for analysis.

264 Whole genome sequencing (WGS) and bioinformatics

265 Based on the qPCR results, DNA from the same samples from nine participants spread across

266 the full range of rDNA gene dosage ( $n=3$ low, $n=3$ middle, $n=3$ high) was selected for WGS. At

267 least $1.5 \mu \mathrm{g}$ of skeletal muscle DNA was used for analysis. To avoid potential amplification bias, 
268 a PCR-free protocol was used for library preparation. We calculated the rDNA copy number

269 using a similar approach as previously described by Gibbons et al. 2014 by calculating relative

270 depth differences between rRNA sequences and the background (whole genome). Reads were

271 trimmed for adapter sequences and low quality (minimum phred score of 20) before aligning to

272 the GRCh37 (hg19) human reference genome assembly using Bowtie2 v 2.3.4.3 with the "--end-

273 to-end" option (Langmead \& Salzberg, 2012; Langmead et al., 2019). Alignment results,

274 produced in random order, were sorted with respect to their genomic positions using the

275 samtools sort function and read depth at each position was computed using samtools depth

276 function. We took advantage of the 45S rRNA sequence on the supercontig GL000220.1 in the

277 GRCh37 (hg19) reference assembly and used this as a surrogate for the consensus rDNA

278 repeat sequence (U13369), as described by Gibbons et al. We found that read depths computed

279 using the supercontig rRNA regions were highly correlated with those computed from using

$280 \mathrm{U} 13369\left(\mathrm{R}^{2}>0.96\right.$, data not shown). This approach precluded the need to generate a custom

281 reference assembly to combine the rDNA sequence with the rest of the genome and ensured

282 assembly version consistency, thereby limiting the confounding effects of the genome assembly

283 version differences on the variability in background read depths among participants. Maximum

284 read depth corresponding to each rRNA coding region (18S, 5.8S, and 28S) were divided by the

285 average read depth for the whole genome to obtain rDNA component dosage, which is an

286 estimate of the number of copies of rDNA in a haploid genome.

\section{Mouse study design}

288 To specifically label myonuclei via genetic means, we generated female $\mathrm{HSA}^{+/-}$-GFP ${ }^{+/-}$mice by

289 crossing homozygous human skeletal actin reverse tetracycline transactivator (HSA-rtTA) mice

290 developed by our laboratory (Iwata et al., 2018) with homozygous tetracycline response element

291 histone 2B green fluorescent protein mice (TetO-H2B-GFP) obtained from the Jackson

292 Laboratory (005104). GFP labeling is $>90 \%$, is highly specific to myonuclei, and does not result

293 in labeling of satellite cell-derived myonuclei during the experimental period (Iwata et al., 2018),

294 thus making the results specific to resident myonuclei. Mice were treated with doxycycline in

295 drinking water $(0.5 \mathrm{mg} / \mathrm{ml}$ with $2 \%$ sucrose $)$ for one week. Following doxycycline treatment and a

296 six day washout, mice underwent bilateral sham surgery (biological duplicate) or synergist

297 ablation mechanical overload (OV) of the plantaris (biological triplicate) as described previously

298 (von Walden et al., 2020a), then were euthanized 72 hours later. The mice in these experiments

299 were $\sim 3$ months of age at the time of surgery, and immunohistochemistry and single fiber

300 imaging for representative images is described in (von Walden et al., 2020a). For the metabolic 
301 RNA labeling experiments, age-matched C57BL/6J mice were subjected to sham and OV by the

302 same surgeon as the HSA-rtTA mice (biological duplicate for sham and triplicate for OV).

303 Mouse in vivo metabolic RNA labeling

304 Five hours prior to tissue harvesting after sham and OV, all mice were pulsed with $2 \mathrm{mg}$ of 5-

305 ethynyl-uridine (EU) dissolved in $200 \mu \mathrm{l}$ of PBS via an intraperitoneal injection, as previously

306 described by our laboratory (Kirby et al., 2016). Muscles were snap frozen in liquid nitrogen upon

307 collection. RNA was extracted using TRIzol reagent (Invitrogen) and DirectZol columns with on

308 column DNAse treatment (Zymo Research). RNA was resuspended in molecular-grade $\mathrm{H}_{2} \mathrm{O}$ and

309 quantified by measuring the optical density (230, 260, and $280 \mathrm{~nm}$ ) with a Nanodrop 1000

310 Spectrophotometer (ThermoFisher Scientific, Wilmington, DE). Nascent (EU-labeled) RNA was

311 purified from $1 \mu \mathrm{g}$ RNA per sample using the commercially available Click-iT Nascent RNA

312 Capture Kit (Life Technologies, Carlsbad, CA). cDNA was generated on 500 ng of total RNA and

313 all EU affinity-purified RNA using the SuperScript VILO cDNA Synthesis Kit (Life Technologies).

314 We assessed relative gene expression in the total RNA and nascent RNA fractions by

315 normalization to EMC7 by the comparative $\mathrm{Ct}\left(2^{-\Delta \Delta \mathrm{Ct}}\right)$ method.

\section{RRBS analysis of human skeletal muscle and mouse myonuclear DNA}

317 DNA isolation from human biopsy samples $(\leq 5 \mathrm{mg})$ and mouse myonuclear samples was carried

318 out according to the detailed protocols of Begue et al. (Begue et al., 2017) and von Walden (Von

319 Walden et al., 2020a), respectively. Briefly, using the QIAamp DNA micro kit (Qiagen), muscle

320 samples or myonuclear pellets were re-suspended in "buffer ATL" supplemented with proteinase

$321 \mathrm{~K}$ overnight at $56^{\circ} \mathrm{C}$. DNA binding to the column was conducted using $1 \mu \mathrm{g}$ of carrier RNA (only

322 for myonuclear samples), and washes and centrifugations were carried out according to the

323 manufacturer's instructions. DNA was eluted in $20 \mu \mathrm{l}$ of molecular grade $\mathrm{H}_{2} \mathrm{O}$ and was stored at

$324-80^{\circ} \mathrm{C}$ until the time of analysis. DNA quality assessment and RRBS was conducted in

325 collaboration with Zymo Research. Quality and concentration were assessed using a Fragment

326 Analyzer (AATI). "Classic" RRBS library preparation was performed by digesting 5 ng of genomic

327 DNA with 30 units of Mspl enzyme (New England BioLabs), and fragments were ligated to pre-

328 annealed adapters containing 5'-methyl-cyotosine. Adapter-ligated fragments $\geq 50$ bp were

329 recovered using the DNA Clean \& Concentrator Kit (Zymo Research, D4003) and bisulfite-

330 treated using the EZ DNA Methylation-Lightning Kit (Zymo Research, D5030). Preparative-scale

331 PCR was performed, and the products were purified again using the Clean and Concentrator kit.

332 Paired-end sequencing was performed using Illumina HiSeq, and sequenced reads from

333 bisulfite-treated libraries were identified using standard Illumina base calling software. 
334 Raw FASTQ files were adapter, filled-in nucleotides, and quality-trimmed using TrimGalore 0.6.4

335 using options for "--rrbs" and "--non_directional” mode retaining reads with minimum quality

336 above phred score of 30. FastQC 0.11.8 was used to assess the effect of trimming and overall

337 quality of the data. A custom genome assembly to interrogate rDNA methylation was generated

338 by adding the consensus rDNA repeat sequences, GenBank U13369.1 (Gonzales and Sylvester,

339 1995) and BK000964.3 (Grozdanov et al., 2003), as a separate chromosome to the human

340 (GRCh38p13) and mouse (GRCm39) reference genome assemblies, respectively. Due to

341 mapping interference from highly similar sequences within the reference assemblies, these

342 sequences were found using Blast by comparing the respective rDNA sequences to the

343 reference assembly followed by masking of these sequences in the genome using N's.

344 Alignment to the custom human and mouse reference genomes was performed using Bismark

345 0.19.0. Methylated and unmethylated read totals for each $\mathrm{CpG}$ site were collected using the

346 Methylation Extractor tool. Methylation levels of each sampled cytosine was estimated as the

347 number of reads reporting a "C", divided by the total number of reads reporting a "C" or "T".

348 Differential methylation analyses were performed using R Bioconductor package, methylSig

349 v1.0.0 (Park et al., 2014), which accounts for both read coverage (minimum set to 5x, as

350 previously described by Begue et al. 2017 and von Walden et al. 2020) and biological variation.

351 Differentially methylated regions were determined with tiling using 100 bp segments and no

352 minimum cutoff for $\mathrm{CpG}$ sites. The mouse data were analyzed using a beta-binomial distribution.

353 The human data were analyzed using a generalized linear model accounting for the interaction

354 between exercise status and time since the last bout and included 3 control participants in the

355 model as a covariate (11 total samples), making for a robust statistical approach. CpG coverage

356 in the human samples at individual sites can vary using RRBS, but in all instances were

357 significant $p$ values and low false discovery rate (FDR) was reported, CpGs in $\geq 5$ participants

358 were present in the dataset. The raw sequencing data were deposited in the NCBI Gene

359 Expression Omnibus database (GSE144774).

360 Murine myonuclear isolations were conducted previously, as described by us (Von Walden et al.,

361 2020a). Briefly, after euthanasia via lethal $\mathrm{CO}_{2}$ asphyxiation and cervical dislocation, plantaris

362 muscles were harvested and processed for myonuclear isolation via fluorescent activated cell

363 sorting (FACS). Muscle was dounce homogenized by hand in a sucrose-based physiological

364 buffer, then the crude nuclear suspension was filtered through a $40 \mu \mathrm{m}$ strainer and spiked with 4

$365 \mu \mathrm{l}$ of propidium iodide (PI, $1 \mathrm{mg} / \mathrm{ml}$ stock), then participanted to FACS analysis. Muscle from a

366 control mouse (sucrose-only treated HSA-GFP) was used to determine background

367 fluorescence, and myonuclei were classified as GFP+/PI+ after elimination of doublets via 
368 forward scatter area versus height (biological duplicate for sham and triplicate for overload) and

369 collected in $15 \mathrm{ml}$ conical tubes. Myonuclear suspensions were pelleted at $500 \mathrm{xg}$ for five

370 minutes using a swinging-bucket rotor prior to DNA isolation.

371 Statistical analysis

372 Data was analyzed using a mixed-effects model in which exercise modality as one factor (EE vs.

373 RE vs. CON) and time of biopsy as another (Pre, $30 \mathrm{~min}, 3 \mathrm{~h}, 8 \mathrm{~h}$ and $24 \mathrm{~h}$ ). Potential relationships

374 among the variables assessed were investigated using one-tailed Pearson's correlation analysis.

375 When significant interactions or main effects were found, post hoc tests were performed using

376 Sidak's method. The level of significance was set at 5\% ( $p<0.05)$, and GraphPad Prism 9.0

377 software (San Diego, CA) was used for statistical analysis. For methylation data, $p$ values $<0.05$

378 were utilized, and false discovery rate (FDR) is reported for differentially methylated CpG sites

379 and regions in humans. Data are presented as means \pm standard error of the mean, unless

380 otherwise denoted. 


\section{RESULTS}

383 Resistance exercise (RE) promotes mTORC1, while endurance exercise (EE) promotes

384 AMPK signaling over the 24-hour time course of recovery in muscle

385 To verify that our chosen exercise modalities caused an increase in canonical signaling

386 associated with exercise adaptation (see Figure 1A for study design), we analyzed the

387 phosphorylation of key proteins involved with EE and RE (namely AMPK and mTORC1

388 signaling). As expected, EE and RE promoted different intramuscular signaling patterns. Only EE

389 induced phosphorylation of AMPK at Thr172 30 minutes post exercise ( $p=0.012$, Figure 1B \& C).

390 RE promoted signaling through mTORC1 as assessed by p70S6K at Thr389, specifically at 30

391 minutes (tendency, $p=0.057$ ) and 8 hours $(p=0.042$, Figure $1 \mathrm{~B}, \mathrm{D}, \& \mathrm{E})$, while $\mathrm{EE}$ did not activate

392 p70S6K (Figure 1D). In the control participants, p-p70S6K was induced at 24 hours after the pre

393 biopsy following breakfast, indicating that food intake stimulates p-p70S6K to a similar extent as

394 food intake combined with exercise 24 hours prior ( $p=0.038$, Figures 2D). Similarly,

395 phosphorylation of rpS6 at Ser240/244 increased mainly following RE, and no significant change

396 was observed in CON or EE. At $30 \mathrm{~min}(p=0.0098)$ and $8 \mathrm{~h}(p=0.0052)$, p-rpS6 was increased in

397 the RE group compared to resting levels. Globally, EE stimulated AMPK while RE stimulated

398 mTORC1 signaling, consistent with the literature (Vissing et al. 2013).

399 Resistance exercise preferentially stimulates ribosome biogenesis and Myc gene

400 expression independent from transcription of RNA Pol l-associated factors

401 We utilized 45S pre-rRNA abundance as a readout of Pol I activity and ribosome biogenesis

402 (Leary \& Huang, 2001). EE caused an acute reduction of Pol I activity that was evident by 30 min

403 post-exercise, and EE was significantly different than RE at both $3 \mathrm{~h}$ and $24 \mathrm{~h}$ ( $p<0.05$, Figure 2A).

404 Ribosome biogenesis was significantly induced by RE at $3 \mathrm{~h}$ and $24 \mathrm{~h}$ post-exercise, indicating an

405 "early" and "late" ribosome biogenesis response. Genes that are responsive to exercise, MSTN,

406 ATROGIN1, and MURF1 (Louis et al. 2007), were generally affected in our time-course, but to a

407 much greater extent with $\mathrm{RE}(p<0.01$, Fig 2B). MSTN was reduced relative to CON 8h after EE

408 and $\operatorname{RE}(p<0.05)$ and was also lower at $3 \mathrm{~h}$ and $24 \mathrm{~h}$ with $\mathrm{RE}(p<0.05)$. ATROGIN1 was elevated

409 at $3 \mathrm{~h}$ and lower at $24 \mathrm{~h}$ with EE $(p<0.05)$, but was lower at $3 \mathrm{~h}$, $8 \mathrm{~h}$, and $24 \mathrm{~h}$ with $\mathrm{RE}(p<0.05)$.

410 There was an effect of time for MURF1, but otherwise no significant changes. Gene expression

411 of factors associated with Pol I regulon formation and Pol I transcription (UBTF, TIF1A, PAF53)

412 were not significantly affected by exercise, and therefore did not match the induction and

413 suppression of 45S pre-rRNA seen following RE and EE, respectively (Figure 2B). Likewise,

414 mRNA abundance of genes involved in rRNA processing (BOP1, NOP56, PES1, and 
415 NUCLEOLIN) were not significantly affected by acute exercise in either modality (Fig 2B), with

416 the exception of a reduction in PES1 30 minutes after $\mathrm{RE}(\mathrm{p}<0.05$, Figure 2B). C-MYC (referred

417 to as MYC for humans and Myc for mice), which is upstream of Pol I regulon formation (von

418 Walden et al., 2012), was $\sim 2$ fold up-regulated in response to RE after 30 min and >15 fold

419 higher after $3 \mathrm{~h}(p<0.05$, Figure 2C). Marked responsiveness of MYC within $24 \mathrm{~h}$ of acute RE in

420 vastus lateralis muscle is supported by meta-analytical data from resistance exercise

421 transcriptome studies (Zierath and Pillon laboratories, www.MetaMEx.eu) spanning 110 healthy

422 young and middle-aged men and women (log fold change=1.48, FDR=0.007x10-4) (Pillon et al.

423 2020); for reference, log fold change for MSTN in the same individuals was -0.81

$424\left(\mathrm{FDR}=0.001 \times 10^{-4}\right)$. Expression levels of all genes in the control condition were not significantly

425 altered at any time point for any gene.

426 Relative rDNA gene dosage is related to rDNA transcription following a bout of resistance 427 exercise

428 Since rDNA copy number can vary widely across people (Gibbons et al., 2014), we hypothesized

429 that relative rDNA dosage would correlate with the ribosome biogenesis response to RE. First, to

430 accurately assess relative rDNA gene dosage in our participants, we ranked all 30 individuals

431 according to their normalized 18S rDNA gene content using a qPCR approach for rDNA

432 quantification (Gibbons et al., 2014). The range of relative rDNA gene dosage among our 30

433 participants displayed a $\sim 3$-fold difference when comparing the lowest versus the highest values

434 (Figure 3A). Based on this ranking, we selected the top three, bottom three, and three individuals

435 in the middle of the distribution and performed whole genome sequencing (WGS) to directly

436 assess rDNA copy number. From the WGS data, we calculated rDNA copy number and

437 observed a significant positive correlation between this value and our QPCR quantification of $18 \mathrm{~S}$

$438(r=0.793, p=0.005$, Figure 3B) and 28S $(r=0.590, p=0.047$, Figure 3C) DNA. Moreover, the $q P C R$

439 quantification of the different coding regions of the rDNA gene showed strong inter-correlation

440 (28S versus 18S, $r=0.846, p<0.0001,28 S$ versus $5.8 S, r=0.975, p<0.0001$, and $18 S$ versus $5.8 S$,

$441 \mathrm{r}=0.862, p<0.0001$, Fig 3D-F), whereas quantification of the 5S rDNA gene (not part of 45S RNA

442 gene) was less correlated with the other rRNA regions (5S versus $28 \mathrm{~S}, \mathrm{r}=0.297, p=0.066,5 \mathrm{~S}$

443 versus $18 \mathrm{~S}, \mathrm{r}=0.386, p=0.023,5 \mathrm{~S}$ versus $5.8 \mathrm{~S}, \mathrm{r}=0.382, p=0.042$, Figure $3 \mathrm{G}-\mathrm{I}$ ). Relative rDNA

444 dosage did not correlate to ribosome biogenesis at rest (data not shown). Finally, the increase in

445 45S pre-rRNA 24 hours after RE was positively correlated with relative rDNA dosage (18S,

$446 \mathrm{r}=0.652, p=0.056 ; 28 \mathrm{~S}, \mathrm{r}=0.729, p=0.0313 ; 5.8 \mathrm{~S}, \mathrm{r}=0.696, p=0.041$, Figures $3 \mathrm{~J}-\mathrm{L}$ ). 
449 The human rDNA promoter encompasses a 174 base pair long section of the 45S RNA gene

450 that include two important elements; the core promotor spanning -45 to +18 and the Upstream

451 core element (UCE) -156 to -107 , both relative to the transcription start site (Haltiner et al., 1986)

452 (Figure 4A). We first used the Agena EpiTYPER, a sensitive and targeted mass spectrometry-

453 based array method to compare the degree of methylation of the rDNA promoter in resting

454 skeletal muscle at five sites within the promoter region. We calculated an average skeletal

455 muscle rDNA promoter methylation of $23 \pm 8 \%$ in resting skeletal muscle (Figure $4 \mathrm{~B}$ ), in

456 agreement with our previous work (Von Walden et al., 2020b), and consistent with levels

457 reported in other human non-muscle tissues (Pietrzak et al., 2011; Uemura et al., 2012).

458 Ribosomal DNA promoter methylation varied from $12 \%$ to $30 \%$ depending on the site, but site-

459 specific methylation on a person-by-person basis tracked well (colored dots), so the average

460 value is reflective of methylation along the promoter (Figure $4 \mathrm{C}$ ). Average methylation was

461 neither related to 45S pre-rRNA levels $(r=-0.17, p=0.41$, Fig 4D) nor total RNA content $(r=-0.14$,

$462 p=0.26$, Fig 4E) at rest. These data collectively suggest that the regulation of ribosome

463 biogenesis in skeletal muscle under resting conditions is not influenced by rDNA promoter

464 methylation nor gene dosage.

465 Resistance exercise acutely modifies methylation of the rDNA gene without affecting the 466 promoter

467 To explore whether the acute modulation of $45 S$ pre-rRNA abundance following EE (down468 regulation) and $\mathrm{RE}$ (up-regulation) was associated with epigenetic modifications at the rDNA 469 promoter, we analyzed targeted rDNA promoter methylation at all time points in all participants 470 via EpiTYPER. We found no changes in average rDNA promoter methylation irrespective of 471 exercise (Figure 5A). Although rDNA promoter methylation was not altered by exercise, the 472 robust induction of ribosome biogenesis and MYC throughout the 24-hour time course following 473 RE provided rationale to characterize methylation of other regulatory regions along the rDNA 474 repeat in greater detail.

475 We conducted RRBS on DNA from a subset of RE participants at pre exercise, 30 minutes post, 476 and 24 hours post exercise $(n=8)$ and included data from 3 non-exercise participants at the 477 corresponding time points in the analysis to account for the effects of biopsy and time. There is a 478 strong interrelationship between chromatin modifications and DNA methylation (Eden et al., 479 1998; Fuks, 2005; Nemeth et al., 2008; Cedar \& Bergman, 2009), so published chromatin 480 immunoprecipitation (ChIP) and rDNA mapping data (Grandori et al., 2005; Zentner et al., 2011a; 
481 Shiue et al., 2014; Zentner et al., 2014; Agrawal \& Ganley, 2018) were used to guide our

482 analysis and infer implications of methylation patterns revealed by RRBS. Of the few CpG sites

483 altered at both time points after RE, site 42523 relative to the transcription start site (TSS) had

484 high coverage across individuals and was hypomethylated at 30 minutes $(-17 \%, p=0.0002$,

485 FDR=0.03) and 24 hours $(-32 \%, p=0.00003$, FDR=0.01) (Figure $5 B)$; this site is in a region

486 characteristic of the rDNA enhancer (Zentner et al. 2011a), defined as H3K4me1/2 enriched

487 (Zentner et al., 2011b). Since Myc mRNA was robustly up-regulated with RE, we looked for

488 methylation differences in rDNA regions where MYC protein may associate. Thirty minutes after

489 RE, an IGS CpG site in an area with MYC binding affinity (Grandori et al. 2005, Agrawal \&

490 Ganley, 2018) was hypomethylated (site 17711, -11\%, $p=0.0005$, FDR=0.08) (Figure 5C).

491 Immediately upstream of a region where MYC is most enriched on rDNA ( 13 kb from the TSS)

492 (Grandori et al. 2005), a CpG site was hypomethylated 30 minutes after RE (site 12054, 98\%

493 versus $88 \%, p=0.0007, F D R=0.09$ ). Human rDNA has a unique enhancer-like region in the IGS

494 (Zentner et al., 2011a) that contains MYC occupancy sites (Agrawal \& Ganley, 2018) and codes

495 for a stress-responsive IGS transcript (IGS 28 RNA) (Audas et al., 2012). Immediately upstream of

496 this region, two highly methylated CpG sites in close proximity (site 27603 and 27614) were both

$497 \sim 30 \%$ hypomethylated 30 minutes after RE ( $p=0.0003$, FDR=0.05 and $p=0.002$, FDR=0.21,

498 respectively) (Figure 5D). Other hypomethylated CpG sites 30 minutes after RE were 4159 and

499 34987, and hypermethylated sites were 11938, 20819, 33349, and 33624 (FDR<0.05). 24 hours

500 after RE, CpG sites 37512 and 39163 were hypomethylated, and 765, 11938, 20819, 33349,

50133806 , and 34428 were hypermethylated (FDR<0.05). Finally, a cluster of hypermethylated CpG

502 sites 30 minutes after RE emerged 4-5 kb downstream of the TSS in the $18 \mathrm{~S}$ coding region

$503(4359,4367,4369,4374$, and 4382) (FDR<0.05).

504 MYC transcription and rDNA methylation changes observed in human biopsy samples

505 with RE were conserved with an acute hypertrophic stimulus in mice

506 Since skeletal muscle is a mixed tissue containing nuclei from various cell types, any epigenetic

507 changes in rDNA genes in myonuclei could be obscured by non-muscle nuclei. Using a novel

508 murine in vivo genetic myonuclear labelling strategy (HSA-GFP) to investigate global DNA

509 methylation changes in response to an acute hypertrophic stimulus, we previously published that

510 the responses are distinct between purified myonuclei and interstitial nuclei (von Walden et al.,

511 2020a). Here, we confirm that 72 hours of mechanical overload (OV) of the plantaris muscle via

512 synergist ablation in mice was associated with significantly higher abundance of the 45S pre-

513 rRNA (Figure 6A). In vivo metabolic labeling of nascent RNA revealed that greater levels of rRNA 
514 was due to de novo synthesis, as illustrated by the elevated abundance of this transcript in the

515 EU-labeled fraction following OV (Figure 6A). Myc was also highly enriched in the EU fraction

516 after 72 hours of OV (Figure 6B). This finding verified that Myc is primarily regulated at the

517 transcriptional level in response to OV, and not by post-transcriptional mechanisms, such as

518 enhanced mRNA stability.

519 To complement the human RRBS data, we gathered detailed information on the epigenetic

520 regulation of rDNA specifically within myonuclei in response to an acute hypertrophic stimulus in

521 mice. Labeled myonuclei from HSA-GFP mice were isolated via FACS (Figure 7A) and

522 myonuclear rDNA methylation in sham and 72-hour OV plantaris muscle was analyzed (Figure

523 7B). DNA methylation patterns were altered in regions throughout the rDNA repeat. We observed

524 differential methylation patterns with OV relative to sham in an rDNA enhancer region $\sim 43-45 \mathrm{~kb}$

525 downstream of the TSS (Zentner et al., 2014), with hypermethylation in the 44501-44600 kb

526 region $(p=0.02, \mathrm{FDR}=0.14)$ and hypomethylation in the $44701-48000 \mathrm{~kb}$ region $(p=0.002$,

527 FDR=0.08) (Figure 7B). In this enhancer region, differential methylation generally occurred at

528 sites with higher levels of methylation ( $>50 \%$ ), and where methylation was $>50 \%$ at either time

529 point, hypomethylation predominated with OV ( $p \leq 0.05$, Figure $7 C)$. There are three major

530 occupancy regions for Myc protein in mouse rDNA according to ChIP-sequencing: canonical

531 binding in the upstream core element/promoter, a site within $1 \mathrm{~kb}$ downstream of the TSS, and in

532 a $\sim 7-13 \mathrm{~kb}$ downstream region (Zentner et al., 2014); differential methylation during OV coincides

533 with the latter two regions (Figure 7A). The $201-300 \mathrm{~kb}(-19 \%)$ and $401-500 \mathrm{~kb}(-10 \%)$ regions

534 downstream of the TSS were hypomethylated with OV $(p=0.01, \mathrm{FDR}=0.12$ and $p=0.02$ and

535 FDR=0.14, respectively), within which specific $\mathrm{CpG}$ sites were significantly hypomethylated

$536(-19 \%$ at site 203 and $-61 \%$ at site $466, p<0.05)$. The 11601-11700 downstream region was

537 hypermethylated during OV $(p=0.02, \mathrm{FDR}=0.14)$ with an individual significantly hypermethylated

538 CpG site therein $(+26 \%$ at site $11674, p<0.05)$. On average, methylation in the $7-13 \mathrm{~kb}$ region

539 was low and slightly hypermethylated with OV ( 23\% versus $27 \%)$, but we observed that in

540 individual differentially methylated CpG sites where methylation was above $50 \%$ at either time

541 point in this region, hypomethylation with OV predominated ( $p \leq 0.05$, Figure 7D). Finally, in the

542 IGS, the 39401-39500 region was hypomethylated after OV $(-9 \%, p=0.03$, FDR=0.14) with one

543 specific CpG site therein trending to be hypomethylated with OV ( $-8 \%$ at $39484, p=0.06)$. 


\section{DISCUSSION}

545 This study reveals that rDNA transcription in response EE or RE is not associated with changes

546 in promoter methylation, but may be influenced by methylation within enhancer, IGS, and MYC

547 binding regions specifically with RE. The human findings are corroborated by myonuclear-

548 specific rDNA methylation after acute OV in mice, indicating a conserved response in murine

549 muscle fibers. rDNA copy number correlates to the ribosome biogenesis response to RE, while

550 the skeletal muscle response to EE seems less reliant on genetic and epigenetic factors

551 regulating rDNA transcription. Ribosome biogenesis is suppressed in response to EE, indicating

552 that rDNA transcription is a feature specific to hypertrophic adaptations, at least within the 24

553 hour time window after an acute bout.

554 To characterize our stimuli at the molecular level, we measured ribosome biogenesis and

555 phosphorylation status of key proteins in signaling transduction pathways known to be

556 responsive to exercise, specifically AMPK and mTORC1. Acute EE may repress ribosome

557 biogenesis (Hansson et al. 2019), and ribosome biogenesis throughout our time course (pre-45S

558 rRNA) was generally repressed by EE but was induced at 3 hours and 24 hours after RE. AMPK

559 signaling was more prominent with EE versus RE, broadly consistent with the literature (Vissing

560 et al. 2013, Murach \& Bagley, 2016). mTORC1 is involved in muscle ribosome biogenesis (von

561 Walden et al., 2016) and p70S6K Thr ${ }^{389}$ is a direct target of mTORC1, known to be highly

562 phosphorylated in response to RE. Phosphorylation of this site was significantly increased in the

563 RE group throughout the time course, but was also stimulated by feeding alone and endurance

564 exercise with feeding at the 24 hour time point, uncoupled from ribosome biogenesis. Our

565 ribosome biogenesis findings generally provide human in vivo support for a hypothesis where

566 mTOR promotes Pol I transcription whereas AMPK inhibits it (von Walden, 2019), but also

567 suggest mechanisms beyond signaling play a role in ribosome biogenesis with acute exercise.

568 Furthermore, changes in transcriptional regulators involved in ribosome biogenesis and rRNA

569 processing were unrelated to changes in 45S pre-rRNA at any time point, whereas the early

570 induction of MYC did coincide with ribosome biogenesis. While robust up-regulation of $M Y C$ is

571 consistent with its role in hypertrophic muscle adaptation (Chaillou et al., 2014; Wen et al., 2016;

572 Figueiredo \& McCarthy, 2019b; von Walden, 2019), the overall signaling and transcriptional

573 findings motivated us to explore alternative regulatory mechanisms for ribosome biogenesis.

574 Skeletal muscle mass regulation has long been hypothesized to possess a strong genetic

575 component (Seeman et al., 1996). While the search for genetic explanations of muscle

576 hypertrophy have largely focused on normal protein-coding genes and gene polymorphisms 
577 (Ivey et al., 2000; Riechman et al., 2004; Charbonneau et al., 2008; Li et al., 2014), rDNA copy

578 number has been overlooked. We first utilized WGS to quantify rDNA copy number in nine select

579 individuals, then correlated the results to a qPCR method using validated primers from a

580 previous study (Gibbons et al., 2015) to confirm the veracity of this approach in our samples. The

581 qPCR method correlated well to the WGS data, supporting qPCR based relative rDNA gene

582 dosage estimation. In our cohort, there was a three-fold difference between individuals with the

583 lowest and the highest relative rDNA dosage, aligning with what has been previously reported

584 (Gibbons et al., 2014b). The available evidence indicates that 45S pre-rRNA levels may peak at

585 24h after RE (Figueiredo \& McCarthy, 2019a). Similar to previous observations, 45S pre-rRNA

586 levels were significantly upregulated at the 24h time point in the RE group (Stec et al., 2015;

587 Figueiredo et al., 2016), and changes in 45S pre-rRNA at 24h after RE were associated with

588 rDNA copy number. An early ribosome biogenesis response to RE (within $3 \mathrm{~h}$ ) could therefore be

589 related to signaling events, while the later response (24h) could be more influenced by the

590 available template (i.e., number of rDNA copies), as opposed to the activation of anabolic

591 signaling transduction pathways. Post-exercise anabolic signaling may therefore be permissive

592 for early activation but not determinant for sustained upregulation of 45S pre-rRNA levels

593 following an acute bout of RE, which points to a genetic predisposition for hypertrophic

594 responsiveness based on rDNA gene dosage.

595 In muscle undergoing hypertrophy, histone modifications at the rDNA promoter coincide with

596 increased rDNA transcription (von Walden et al., 2012). In light of this epigenetic regulation of

597 rDNA during muscle cell growth, and in context with prior studies reporting genome-wide

598 promoter methylation changes with acute exercise (Barres et al., 2012; Sharples et al., 2016;

599 Seaborne et al., 2018; Sharples \& Seaborne, 2019; Turner et al., 2019; Maasar et al. 2020), we

600 hypothesized that CpG methylation changes to the rDNA promoter region may associate with the

601 response to RE in muscle; however, this was not the case. rDNA promoter methylation in our

602 adult muscle samples was low at rest ( $20 \%$ on average), consistent with our previous report in

603 children (von Walden et al., 2020b), suggesting the promoter is generally available for

604 transcription in a CpG methylation context, and this does not change with acute EE or RE.

605 In addition to promoters, various regulatory elements such as enhancer regions and non-

606 canonical transcription factor binding regions may also influence rDNA transcription (Zentner et

607 al., 2011a; Shiue et al., 2014; Zentner et al., 2014). Hypermethylation of rDNA enhancer regions

608 generally signifies an inactive gene (Brock \& Bird, 1997; Stancheva et al., 1997).

609 Hypomethylation of rDNA enhancer sites in humans and mice after a hypertrophic stimulus, as 
610 well as near a presumed IGS enhancer site/transcript coding region (Audas et al., 2012; Zentner

611 et al., 2011a) suggests epigenetic remodeling of the rDNA repeat may support rDNA

612 transcription in muscle fibers in response to exercise. MYC, a universal amplifier of expressed

613 genes (Nie et al., 2012; Nie et al., 2020), is an rDNA-associated transcription factor that is central

614 to ribosome biogenesis (Arabi et al., 2005), protein synthesis (Van Riggelen et al., 2010; West et

615 al., 2016), and growth (Kim et al., 2000; Xiao et al., 2001; Zhong et al., 2006). MYC localizes in

616 myonuclei during development and hypertrophy (Alway, 1997; Veal \& Jackson, 1998), and its

617 DNA binding extends beyond canonical E-box motifs (Allevato et al. 2017, Guo et al. 2014, Nie

618 et al. 2012 and 2020) and is inhibited by CpG methylation (Prendergast et al., 1991; Perini et al.,

619 2005). A site in the promoter of Myc is hypomethylated after 72 hours of OV in mouse myonuclei

620 (von Walden et al., 2020a), which corresponds with Myc transcription observed here, and

621 progressive Myc protein accumulation after RE in human and rodent muscle (Apró et al. 2013,

622 Figueiredo et al. 2016, Ogasawara et al. 2016). We speculate that altered methylation of MYC-

623 associated areas in rDNA is one component of a coordinated epigenetic regulatory network

624 involving MYC upregulation, sustained ribosome biogenesis, and hypertrophic adaptation in

625 skeletal muscle. There is also a functional link between dynamic MYC binding to IGS rDNA,

626 rearrangement of nuclear architecture, and rDNA transcription specifically during growth in

627 human cells (Shiue et al., 2014). The ramifications of rDNA methylation changes throughout the

628 IGS during muscle hypertrophy in mice and humans deserve further investigation, especially in

629 light of unique functional roles for transcripts originating from the rDNA IGS (Mayer et al., 2006;

630 Santoro et al., 2010; Audas et al., 2012; Vacík et al., 2019). Further inquiry into the

631 consequences and stability of repeat-wide rDNA methylation with exercise will broaden the

632 emerging area of epigenetic regulation of rDNA transcription in skeletal muscle.

633 The induction of rDNA transcription induced by RE is positively associated with rDNA gene

634 dosage in humans, suggesting that rDNA copy number may be a genetic determinant of muscle

635 adaption in response to RE. Given the large variability of rDNA copy number across individuals,

636 and that the muscle hypertrophy response to exercise is highly heterogeneous (Churchward-

637 Venne et al., 2015; Ahtiainen et al., 2016), we propose that rDNA dosage is a potential genetic

638 factor that relates to skeletal muscle hypertrophy induced by resistance training. Acute RE also

639 promotes a reorganization of rDNA methylation patterns along the repeat. The specific

640 consequences and lasting effects of this acute epigenetic remodeling of rDNA deserve further

641 investigation, but if persistent over time, could contribute to a previously observed epigenetic

642 "epi-memory" of prior exercise exposure that facilitates future muscle adaptability (Sharples et

643 al., 2016; Seaborne et al., 2018; Moberg et al., 2020; Murach et al., 2020; Snijders et al., 2020). 


\section{Figure Legends}

645 Figure 1. Exercise-related signaling in response to acute endurance exercise (EE) and

646 resistance exercise (RE) over a 24-hour time course in human skeletal muscle

647 A. Experimental study design timeline illustrating exercise and muscle biopsy collection in EE,

$648 \mathrm{RE}$, and control (CON) participants ( $\mathrm{n}=10$ per group) before (pre), 30 minutes, 3 hours, 8 hours,

649 and 24 hours after exercise

650 B. Western blot images for exercise-responsive protein targets in CON, EE, and RE

651 C. Quantification of phosphorylated AMPK in CON, EE, and RE

652 D. Quantification of phosphorylated p70S6K in CON, EE, and RE

653 E. Quantification of phosphorylated rpS6 in CON, EE, and RE

$654{ }^{*} p<0.05$, Mean \pm SE

656 Figure 2. Transcriptional responses to acute endurance exercise (EE) and resistance exercise

657 (RE) over a 24 hour time course in human skeletal muscle

658 A. RNA Polymerase I (Pol I) transcription of ribosomal rDNA, as assessed by $45 \mathrm{~S}$ pre-rRNA

659 transcription levels, in control (CON), EE, and RE participants

660 B. Heatmap illustrating gene expression for exercise-responsive genes (MSTN, ATROGIN1,

661 MURF1), Pol I transcription factors (UBFT, TIF-1A, PAF53), and 45S pre-rRNA processing

662 factors (BOP1, NOP56, PES1, NUCLEOLIN) in CON, EE, and RE participants

663 C. MYC gene expression in CON, EE, and RE participants, normalized to Pre levels

$664{ }^{*} \&^{* *} p<0.05 \& p<0.01$ compared to CON, respectively; $\dagger p<0.05$ compared to $\mathrm{RE} ; \ddagger p<0.05$

665 compared to CON, Mean \pm SE

667 Figure 3. rDNA dosage for all participants at rest and its association to ribosome biogenesis in 668 response to acute resistance exercise (RE)

669 A. Relative rDNA dosage determined via q-PCR for all participants

670 Comparison of rDNA copy number determined by whole genome sequencing (WGS) relative to

671 rDNA copy number estimated by quantitative PCR (qPCR) using B. 18S rDNA and C. 28S rDNA 
672 Using the qPCR approach for relative DNA dosage determination, relationships between

673 different locations in the $45 S$ gene: D. $28 S$ versus $18 S$, E. $5.8 S$ versus $28 S$, and F. $5.8 S$ versus

674 18S, as well as relationships between G. 5S (not part of 45S) and 18S H. 5S and 28S, and I. 5S

675 and $5.8 S$

676 Relationships between ribosome biogenesis (measured as 45S pre-rRNA) and estimated relative

677 rDNA dosage using J. 18S, K. 28S, and L. 5.8S determined using qPCR

679 Figure 4. rDNA promoter methylation at rest measured via massARRAY EpiTYPER

680 A. Illustration of specific CpG sites in the promoter region of rDNA that were measured in this 681 study. Sites 2 and 4 were combined due to them having the same molecular weight

682 B. Average rDNA promoter CpG methylation levels across all sites measured. Different colors 683 represent the same participants across sites

684 C. rDNA CpG methylation levels at individual sites in the promoter region

685 D. Relationship between ribosome biogenesis and percent promoter methylation

686 E. Relationship between RNA concentration (ng per mg of tissue) and percent CpG promoter 687 methylation

689 Figure 5. rDNA methylation in response to acute resistance exercise (RE)

690 A. Promoter CpG methylation measured via massARRAY EpiTYPER in control (CON), EE, and

691 RE participants over a 24-hour time course in human skeletal muscle

692 B. CpG methylation at a site in the rDNA enhancer region pre, 30 minutes, and 24 hours after

$693 \mathrm{RE}$

694 C. CpG methylation at a site in the rDNA associated with intergenic spacer (IGS) MYC

695 occupancy pre, 30 minutes, and 24 hours after RE

696 D. CpG methylation at sites in the IGS that is enhancer-like and immediately upstream of an

697 IGS-derived transcript

$698 \quad{ }^{* *} \mathrm{FDR}<0.05,{ }^{\#} \mathrm{FDR}=0.05,{ }^{*} p<0.05,{ }^{\$} \mathrm{FDR}=0.21$

699 
700 Figure 6. Ribosome biogenesis and Myc levels in acutely (72 hour) overloaded (OV) plantaris

701 muscle with metabolic RNA labeling

702 A. Total and metabolically labeled (nascent) rRNA in sham and $72 \mathrm{~h}$ OV muscle

703 B. Myc levels in the nascent RNA fraction in sham versus OV muscle

$704{ }^{*} p<0.05$, Mean \pm SE

705

706 Figure 7. Myonuclear-specific rDNA methylation in acutely overloaded (OV) mice measured via

707 reduced representation bisulfite sequencing (RRBS)

708 A. Experimental study design illustrating the treatment and OV of HSA-GFP mice, myonuclear-

709 specific GFP labeling, and isolation of highly-purified GFP+ myonuclei via fluorescent activated

710 cell sorting

711 B. Myonuclear rDNA CpG methylation in sham and 72-hour OV plantaris muscle. rDNA repeat is

712 depicted above the graph based on CpG coverage.

713 C. $\mathrm{CpG}$ methylation in the murine rDNA enhancer region (sites with $>50 \%$ methylation)

714 D. CpG methylation in murine rDNA Myc occupancy region (sites with $>50 \%$ methylation)

$715{ }^{*} p<0.05$ 
bioRxiv preprint doi: https://doi.org/10.1101/2020.12.14.422642; this version posted December 14, 2020. The copyright holder for this preprint (which was not certified by peer review) is the author/funder, who has granted bioRxiv a license to display the preprint in perpetuity. It is made

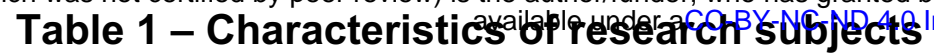

Control

(CON)

Age (yrs)

Sex (male/female)

Height $(\mathrm{cm})$

Weight $(\mathrm{kg})$

Body mass index $\left(\mathrm{BMl}, \mathrm{kg} / \mathrm{m}^{2}\right)$

$\mathrm{VO}_{2 \max }(\mathrm{I} / \mathrm{min})$

$\mathrm{VO}_{2 \max }(\mathrm{ml} / \mathrm{min} / \mathrm{kg})$

Knee extension $(\mathrm{kg})$

Leg press $(\mathrm{kg})$ groups.
$30.5 \pm 8.3$

$6 / 4$

$178 \pm 8.2$

$81.4 \pm 15.5$

$25,5 \pm 4.0$

$3.57 \pm 0.8$

$44.7 \pm 8.8$

$64.0 \pm 17.0$

$142 \pm 40.3$
Resistance

Exercise (RE)

Exercise (EE)

$27.8 \pm 6.8$

$6 / 4$

$176 \pm 10.5$

$78.7 \pm 17.0$

$25.2 \pm 4.7$

$3.62 \pm 0.9$

$47.1 \pm 11.2$

$72.5 \pm 23.2$

$152.6 \pm 55.8$
$33.3 \pm 7.2$

$6 / 4$

$180 \pm 8.9$

$81.2 \pm 9.9$

$24.9 \pm 2.0$

$3.58 \pm 0.9$

$44.1 \pm 9.2$

$68.5 \pm 16.7$

$149.5 \pm 42.7$ 
A

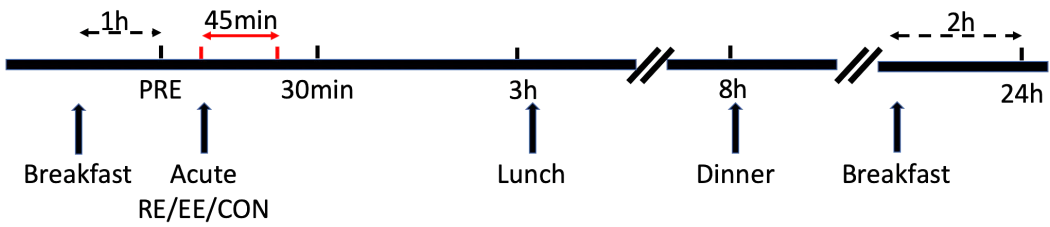

B
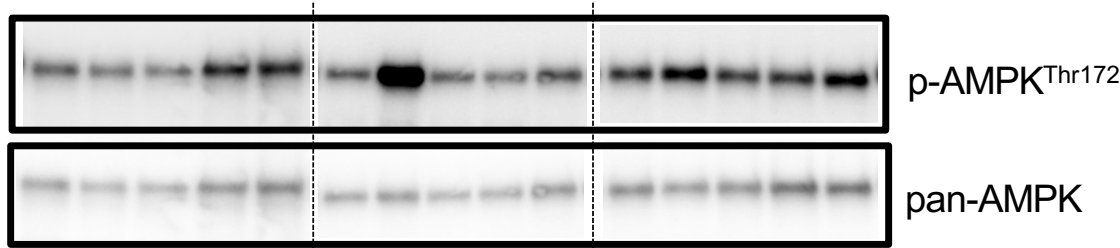

pan-AMPK

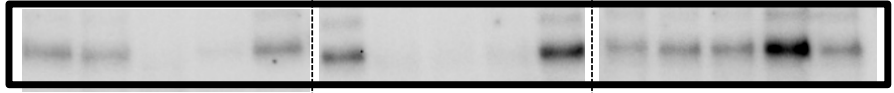

p70S6K Thr389

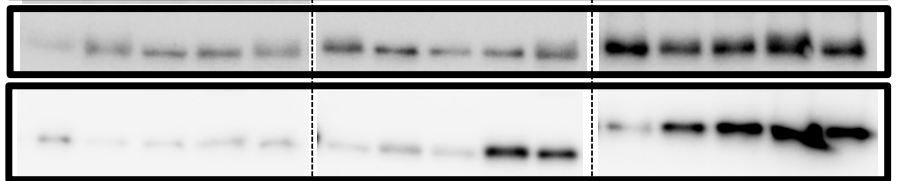

pan-p70S6K

p-RPS6Ser240/244

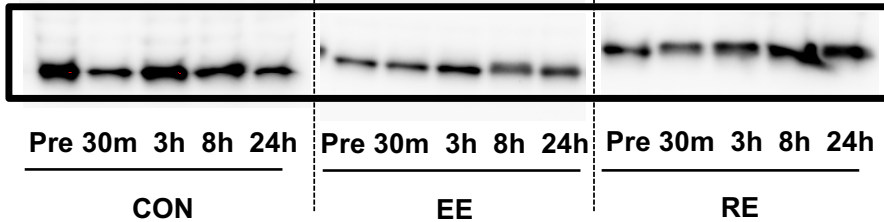

C

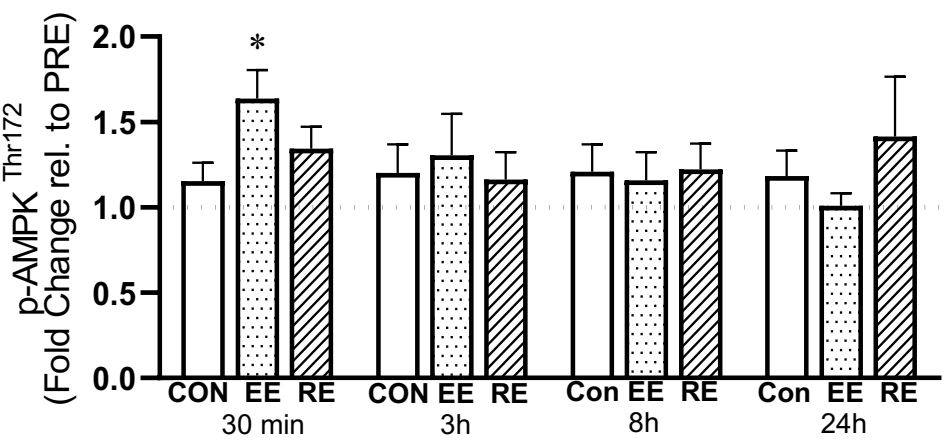

D

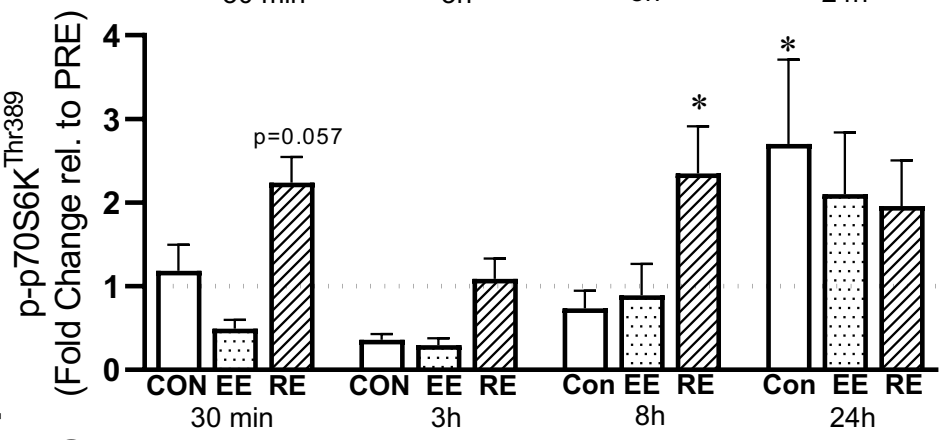

E

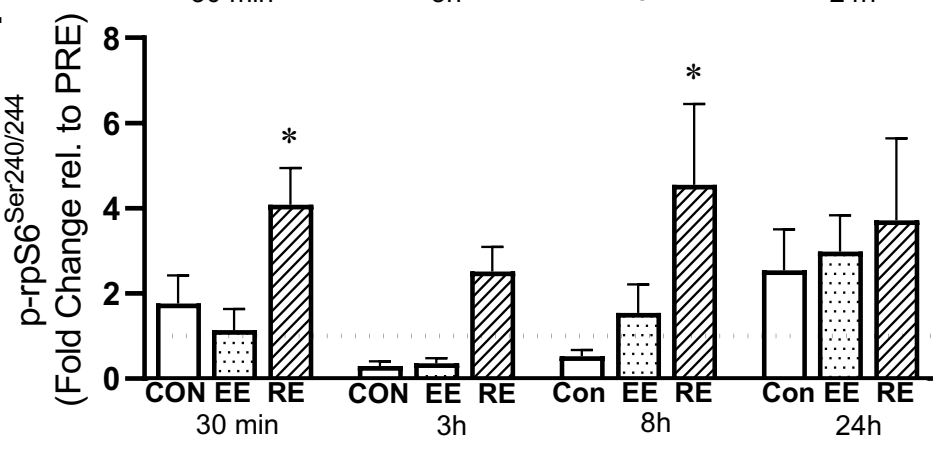


A

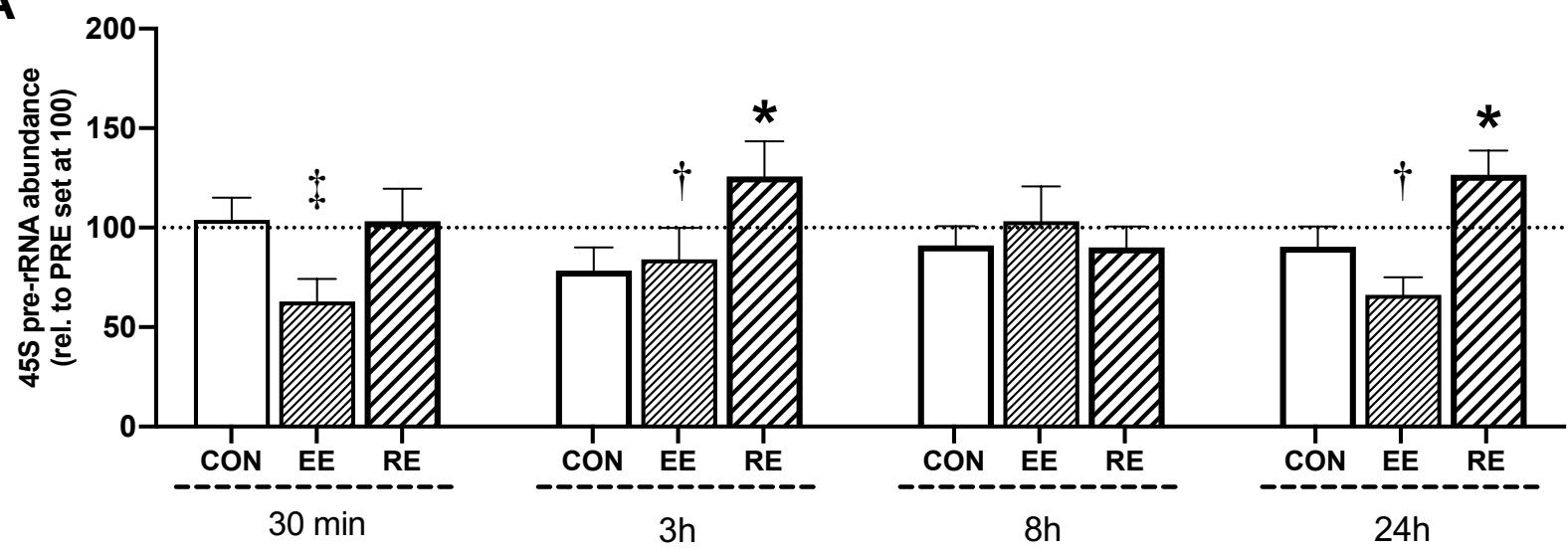

B
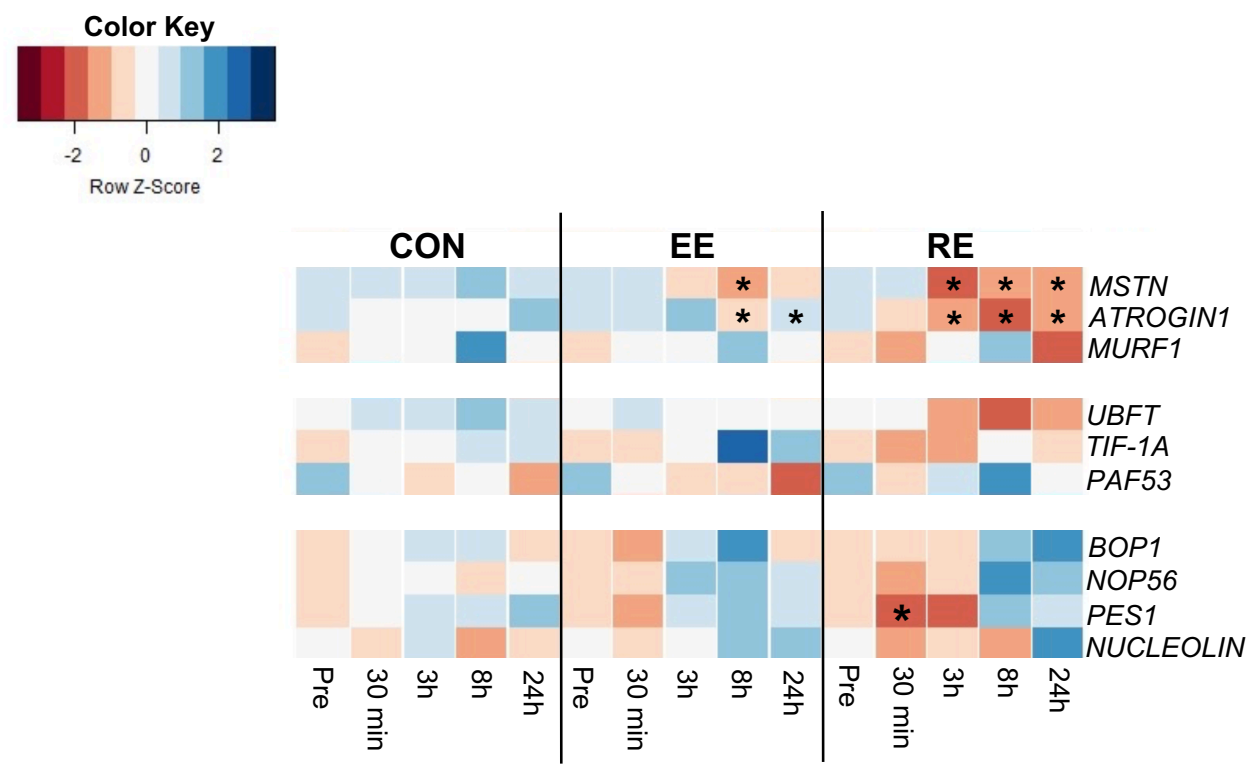

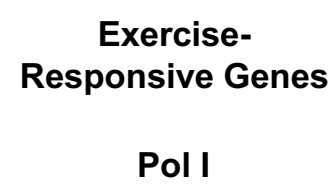

Transcription Factors

\section{S pre-rRNA} Processing Factors

C

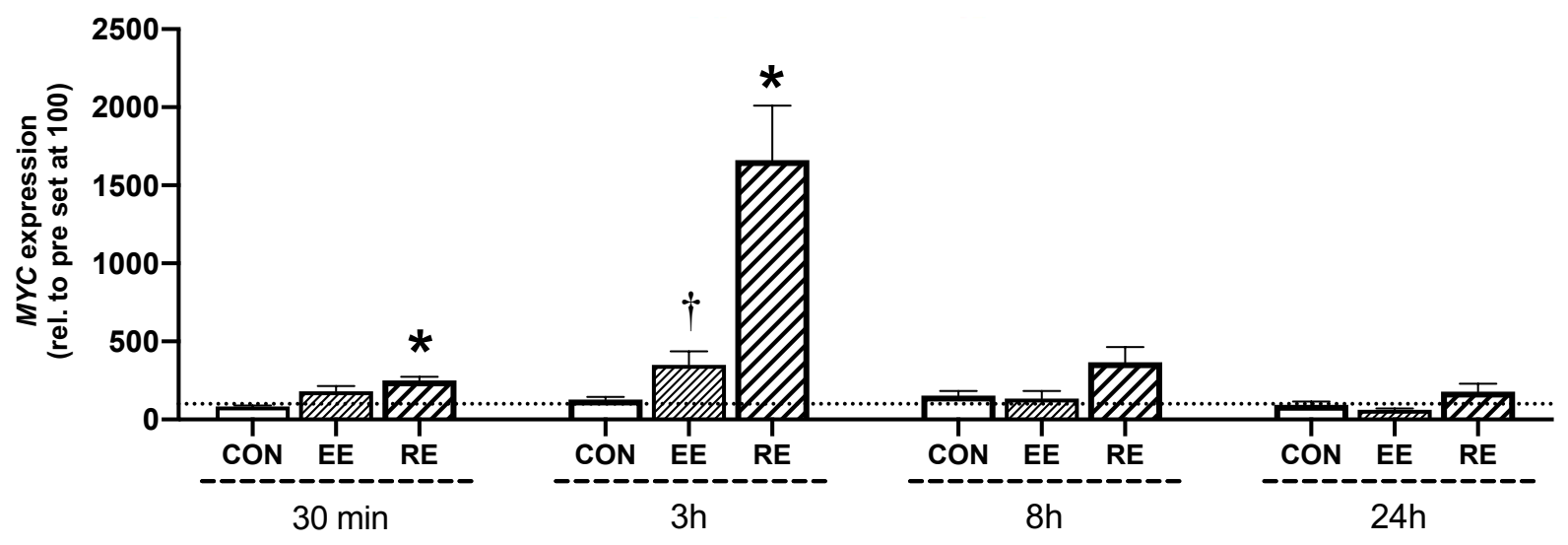


A

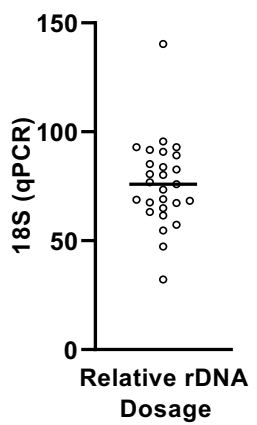

B

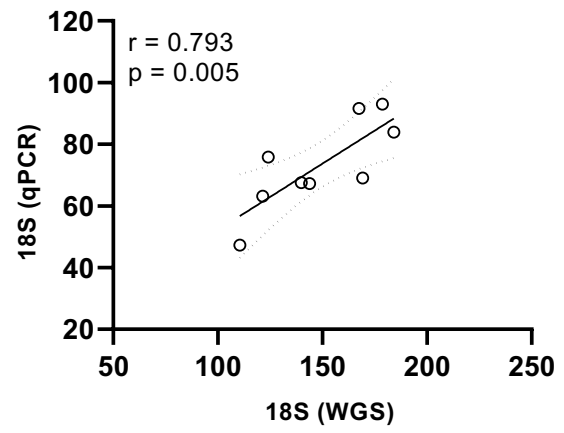

C

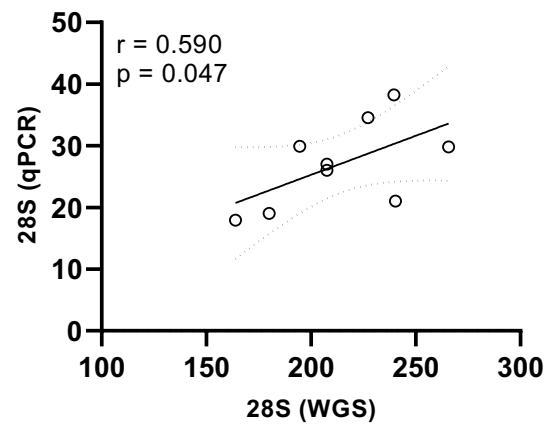

All Subjects

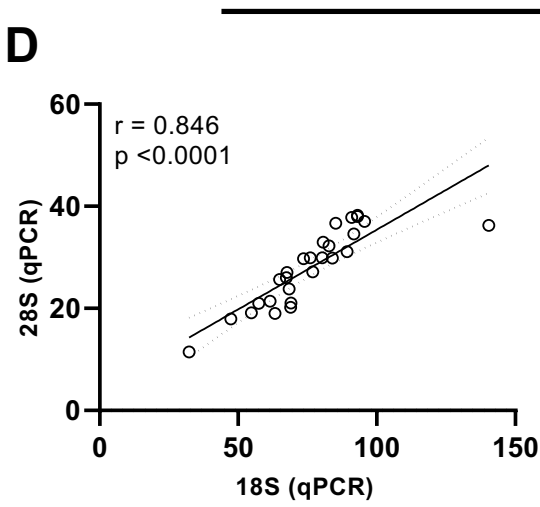

E

F

G
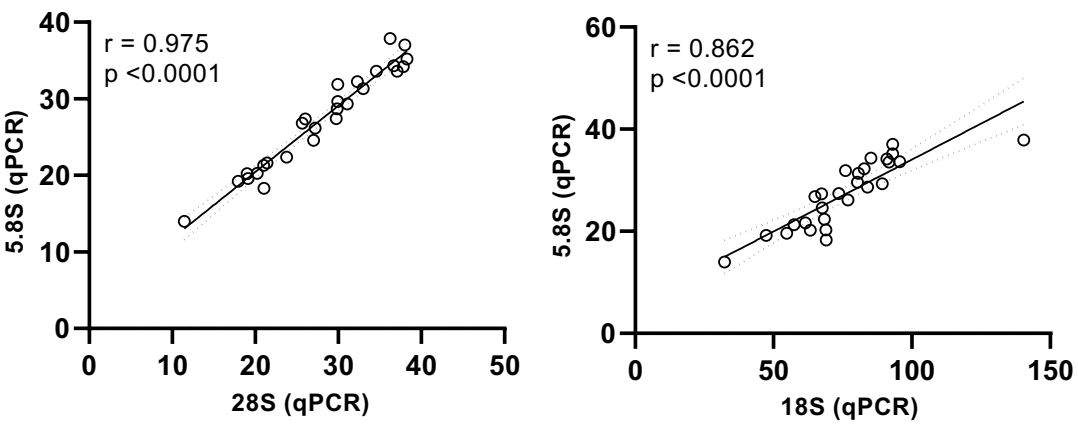

H
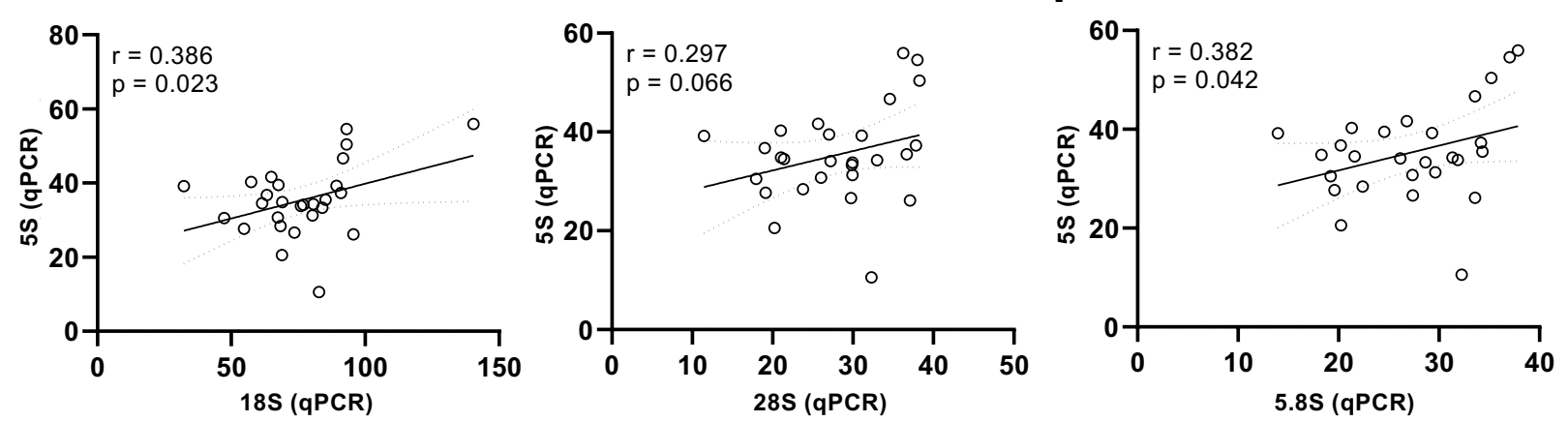

Acute RE (24h)
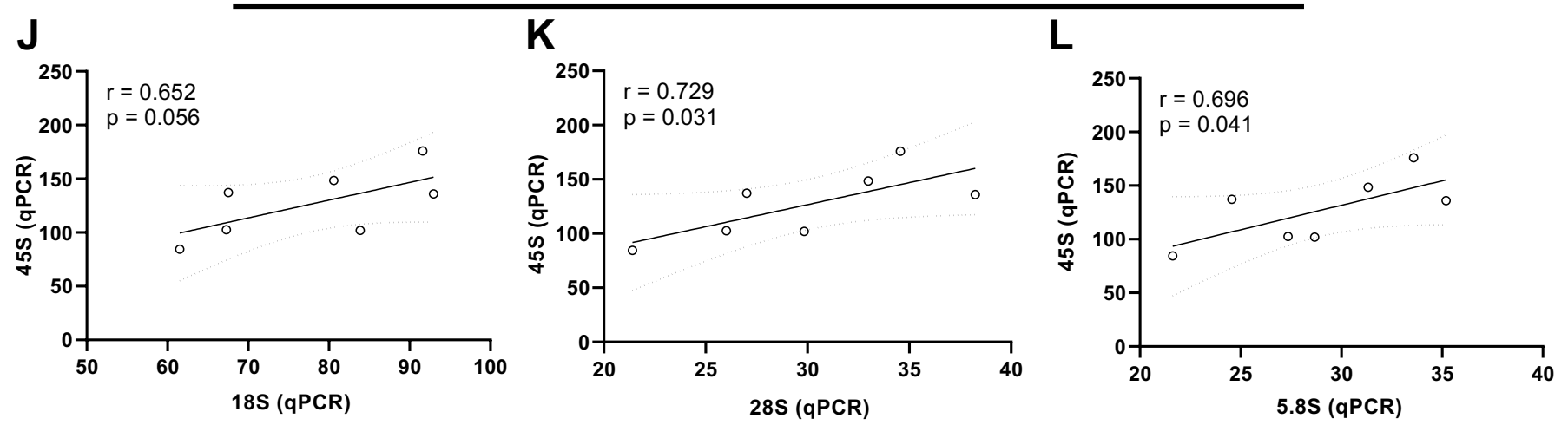
Figure 4

A

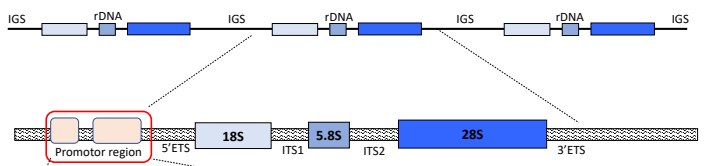

C

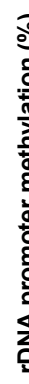

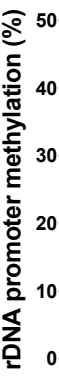

D

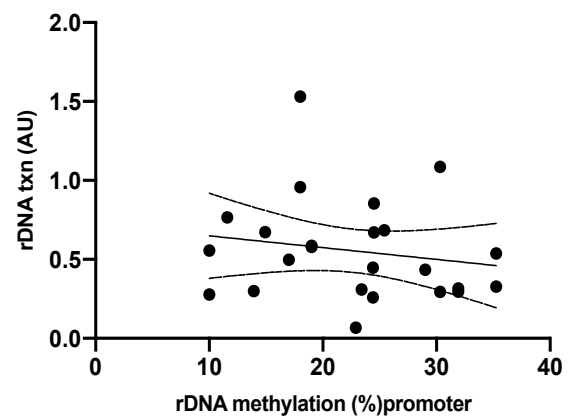

E
B

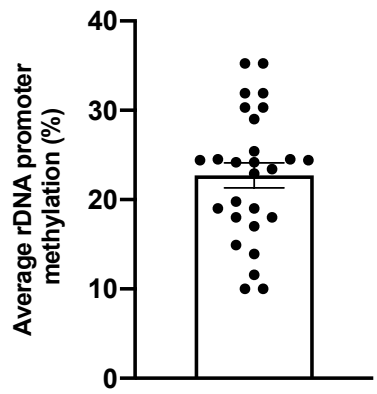

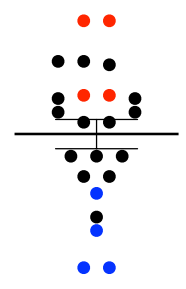

Site 4

Site 5

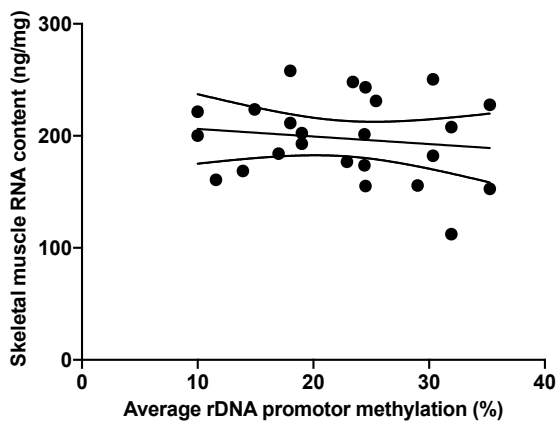


Figure 5

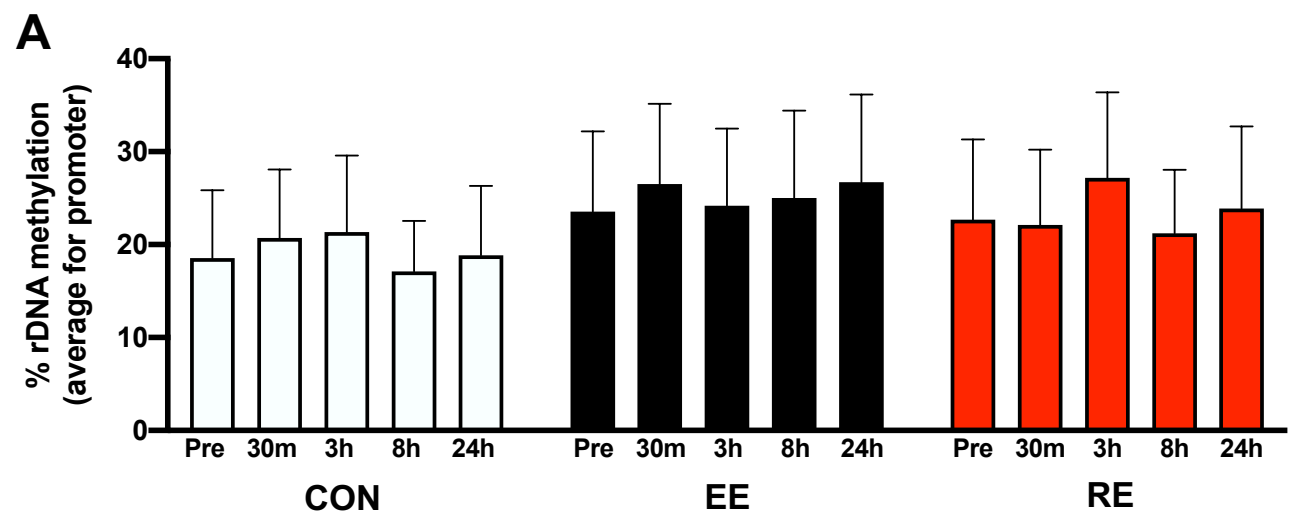

B

Enhancer

C MYC-associated IGS

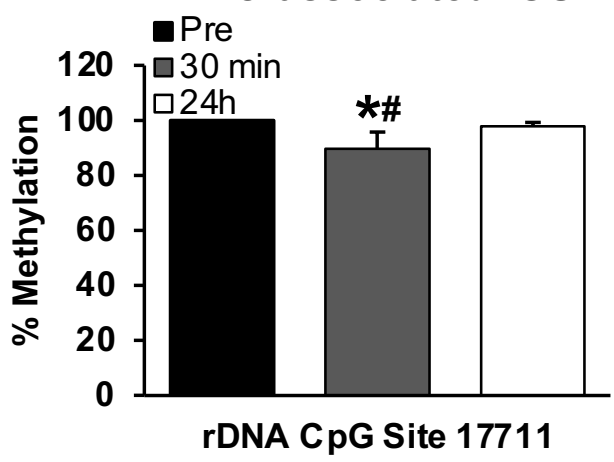

D

IGS
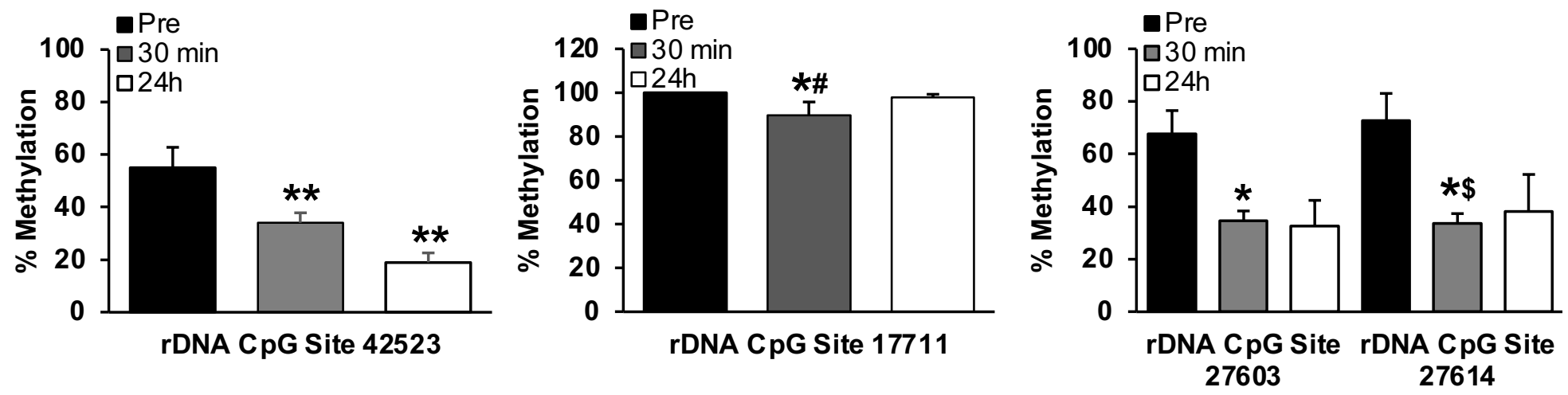
Figure 6

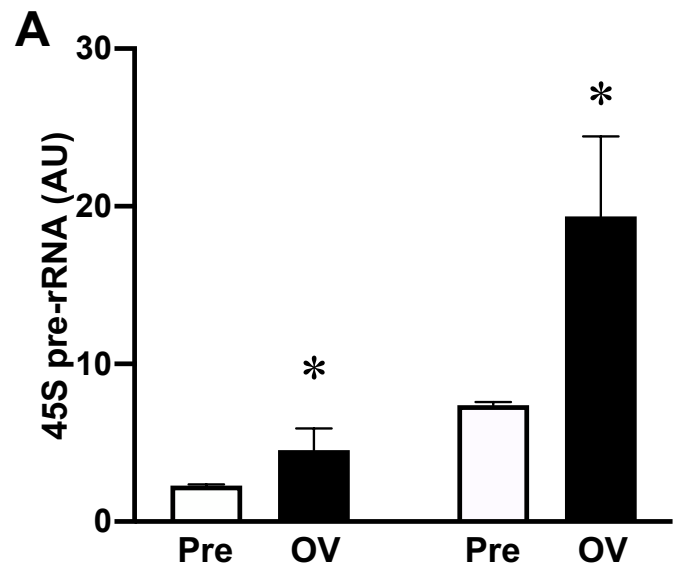

B

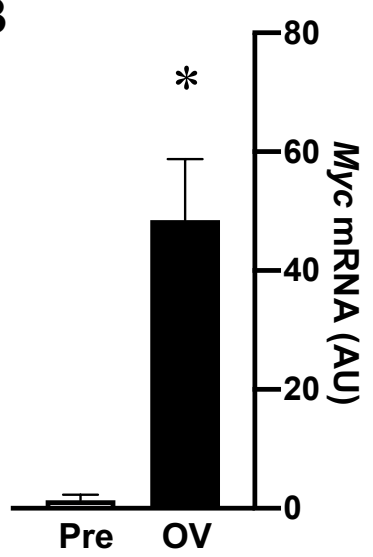

Total RNA

Nascent RNA

Nascent RNA 
Figure 7

A

$6 \mathrm{~d}$

Washout $X$

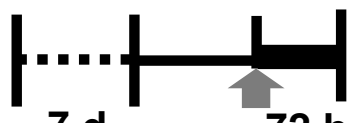

$7 \mathrm{~d}$

$72 \mathrm{~h}$

Doxycycline Sham/OV

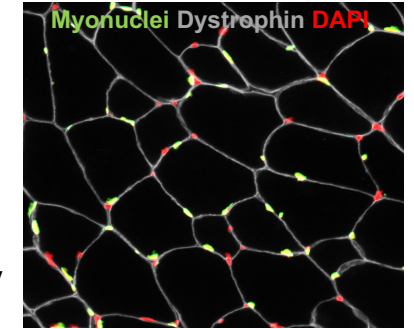

$5.8 \mathrm{~S}$

14-38 kb variable coverage

Intergenic Spacer

43-45kb

Enhancer

Region

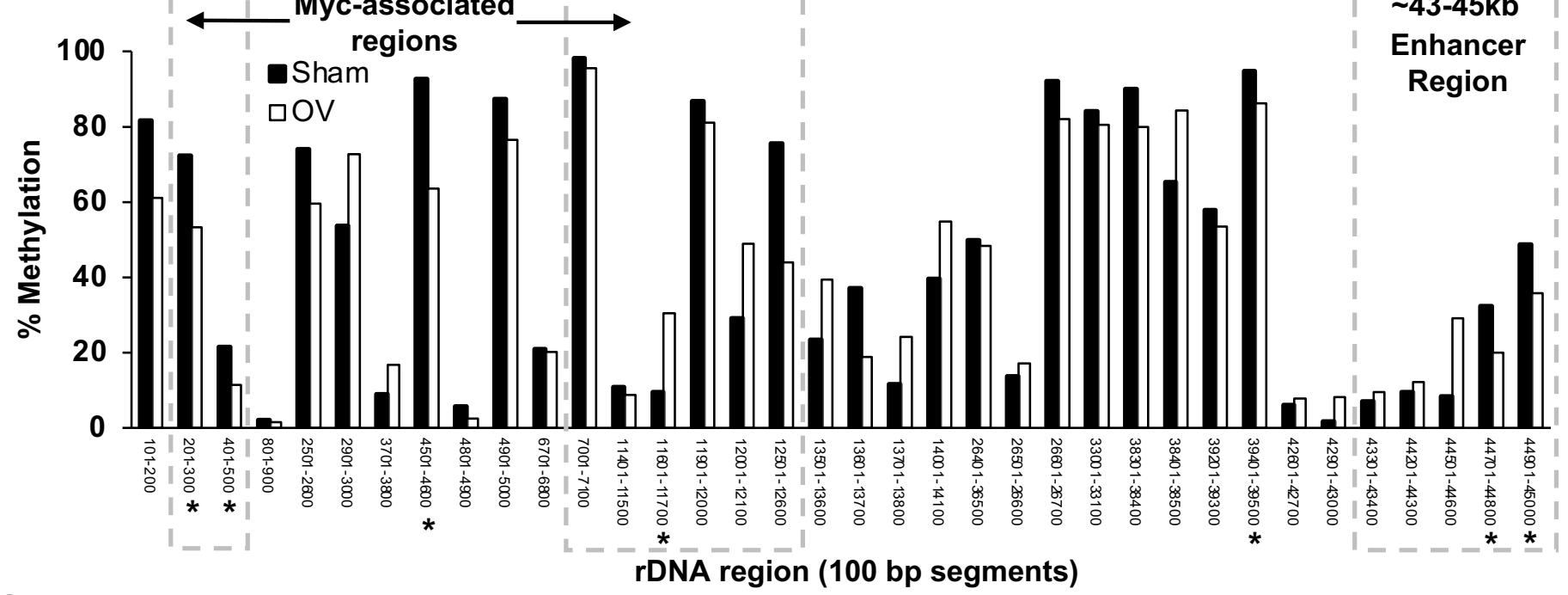

C

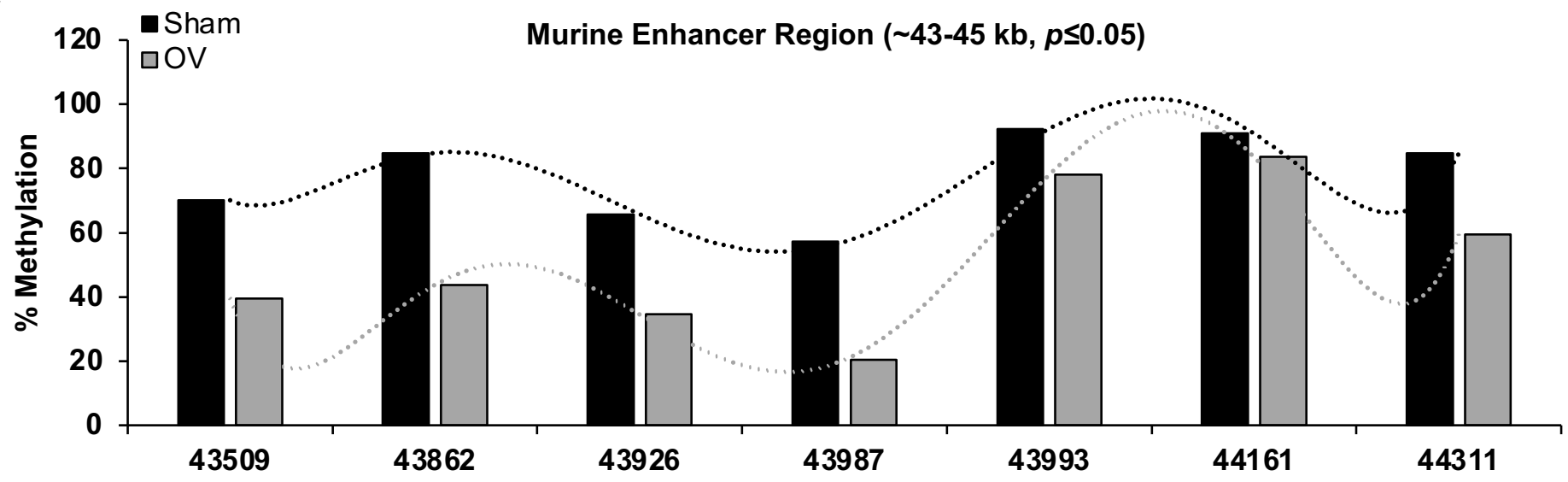

D

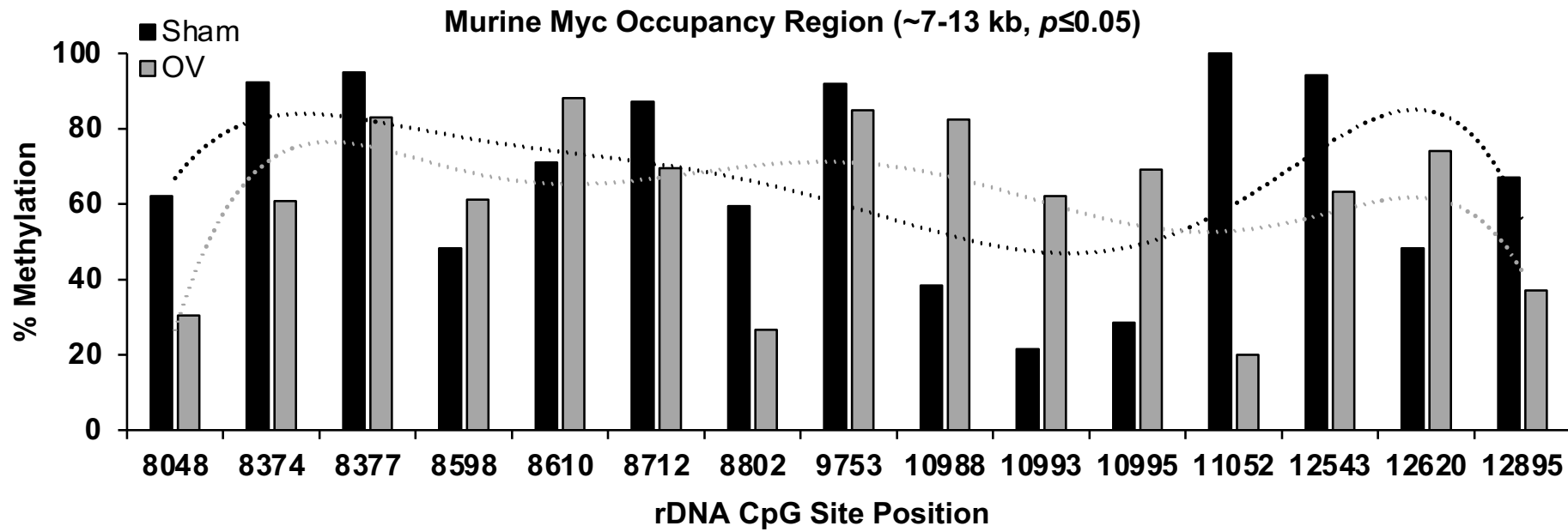




\section{References}

Agrawal S \& Ganley AR. (2018). The conservation landscape of the human ribosomal RNA gene repeats. PloS One 13, e0207531.

Ahtiainen JP, Walker S, Peltonen H, Holviala J, Sillanpää E, Karavirta L, Sallinen J, Mikkola J, Valkeinen H \& Mero A. (2016). Heterogeneity in resistance training-induced muscle strength and mass responses in men and women of different ages. Age 38, 10.

Alway SE. (1997). Overload-induced C-Myc oncoprotein is reduced in aged skeletal muscle. J Gerentol Ser A 52, B203-B211.

Allevato M, Bolotin E, Grossman M, Mane-Padros D, Sladek FM, \& Martinez E. (2017). Sequence specific binding by MYC/MAX to low-affinity non-E-box motifs. PloS One 12, e0180147.

Apró W, Wang L, Pontén M, Blomstrand E \& Sahlin K. Resistance exercise induced mTORC1 signaling is not impaired by subsequent endurance exercise in human skeletal muscle. (2013). Am J Physiol Endo Metab. 305, E22-32.

Arabi A, Wu S, Ridderstråle K, Bierhoff H, Shiue C, Fatyol K, Fahlén S, Hydbring P, Söderberg O \& Grummt I. (2005). c-Myc associates with ribosomal DNA and activates RNA polymerase I transcription. Nat Cell Biol 7, 303-310.

Audas TE, Jacob MD \& Lee S. (2012). Immobilization of proteins in the nucleolus by ribosomal intergenic spacer noncoding RNA. Mol Cell 45, 147-157.

Baldridge GD, Dalton MW \& Fallon AM. (1992). Is higher-order structure conserved in eukaryotic ribosomal DNA intergenic spacers? J Mol Evo 35, 514-523.

Barres R, Yan J, Egan B, Treebak JT, Rasmussen M, Fritz T, Caidahl K, Krook A, O'Gorman DJ \& Zierath JR. (2012). Acute exercise remodels promoter methylation in human skeletal muscle. Cell Metab 15, 405-411.

Begue G, Raue U, Jemiolo B \& Trappe S. (2017). DNA methylation assessment from human slow-and fast-twitch skeletal muscle fibers. J Appl Physiol 122, 952-967.

Bjorkman F, Ekblom-Bak E, Ekblom O \& Ekblom B. (2016). Validity of the revised Ekblom Bak cycle ergometer test in adults. Eur J Appl Physiol 116, 1627-1638.

Borg G. (1970). Perceived exertion as an indicator of somatic stress. Scand J Rehabil Med 2, 9298.

Brock GJ \& Bird A. (1997). Mosaic methylation of the repeat unit of the human ribosomal RNA genes. Human Molec Gene 6, 451-456.

Camelon KM, Hadell K, Jamsen PT, Ketonen KJ, Kohtamaki HM, Makimatilla S, Tormala ML \& Valve RH. (1998). The Plate Model: a visual method of teaching meal planning. DAIS Project Group. Diabetes Atherosclerosis Intervention Study. J Am Diet Assoc 98, 11551158. 
Cedar H \& Bergman Y. (2009). Linking DNA methylation and histone modification: patterns and paradigms. Nat Rev Gene 10, 295-304.

Chaillou T, Kirby TJ \& McCarthy JJ. (2014). Ribosome biogenesis: emerging evidence for a central role in the regulation of skeletal muscle mass. J Cell Physiol 229, 1584-1594.

Charbonneau DE, Hanson ED, Ludlow AT, Delmonico MJ, Hurley BF \& Roth SM. (2008). ACE genotype and the muscle hypertrophic and strength responses to strength training. Med Sci Sports Exerc 40, 677-683.

Churchward-Venne TA, Tieland M, Verdijk LB, Leenders M, Dirks ML, de Groot LCPGM \& van Loon LJC. (2015). There are no nonresponders to resistance-type exercise training inolder men and women. J Am Med Dir Assoc 16, 400-411.

D'Aquila P, Montesanto A, Mandala M, Garasto S, Mari V, Corsonello A, Bellizzi D \& Passarino G. (2017). Methylation of the ribosomal RNA gene promoter is associated with aging and age-related decline. Aging Cell 16, 966-975.

Eden S, Hashimshony T, Keshet I, Cedar H \& Thorne A. (1998). DNA methylation models histone acetylation. Nature 394, 842-842.

Ekblom-Bak E, Bjorkman F, Hellenius ML \& Ekblom B. (2014). A new submaximal cycle ergometer test for prediction of $\mathrm{VO}_{2} \max$. Scand J Med Sci Sports 24, 319-326.

Figueiredo VC. (2019a). Revisiting the roles of protein synthesis during skeletal muscle hypertrophy induced by exercise. Am J Physiol Reg Integr Comp Physiol 317, R709R718.

Figueiredo VC, Caldow MK, Massie V, Markworth JF, Cameron-Smith D \& Blazevich AJ. (2015). Ribosome biogenesis adaptation in resistance training-induced human skeletal muscle hypertrophy. Am J Physiol Endo Metab 30, E72-E83.

Figueiredo VC, Englund DA, Vechetti IJ, Jr., Alimov A, Peterson CA \& McCarthy JJ. (2019). Phosphorylation of eukaryotic initiation factor $4 \mathrm{E}$ is dispensable for skeletal muscle hypertrophy. Am J Physio Cell Physiol 317, C1247-C1255.

Figueiredo VC \& McCarthy JJ. (2019b). Regulation of ribosome biogenesis in skeletal muscle hypertrophy. Physiology 34, 30-42.

Figueiredo VC, Roberts LA, Markworth JF, Barnett MPG, Coombes JS, Raastad T, Peake JM \& Cameron-Smith D. (2016). Impact of resistance exercise on ribosome biogenesis is acutely regulated by post-exercise recovery strategies. Physiol Rep 4.

Fuks F. (2005). DNA methylation and histone modifications: teaming up to silence genes. Curr Opin Gene Devel 15, 490-495.

Gibbons JG, Branco AT, Godinho SA, Yu S \& Lemos B. (2015). Concerted copy number variation balances ribosomal DNA dosage in human and mouse genomes. Proc Nat Acad Sci 112, 2485-2490. 
816

817

818

819

820

821

822

823

824

825

826

827

828

829

830

831

832

833

834

835

836

837

838

839

840

841

842

843

844

845

846

847

848

849

850

851

852

853

854

855

856

857

858

859

860

861

862

863

864

Gibbons JG, Branco AT, Yu S \& Lemos B. (2014a). Ribosomal DNA copy number is coupled with gene expression variation and mitochondrial abundance in humans. Nat Comm 5, 112.

Gonzalez IL \& Sylverster JE. Complete sequence of the 43-kb human ribosomal DNA repeat: analysis of the intergenic spacer. (1995). Genomics, 27, 320-328.

Grandori C, Gomez-Roman N, Felton-Edkins ZA, Ngouenet C, Galloway DA, Eisenman RN \& White RJ. (2005). c-Myc binds to human ribosomal DNA and stimulates transcription of rRNA genes by RNA polymerase I. Nat Cell Biol 7, 311-318.

Grozdanov P, Georgiev O \& Karagyozov L. (2003). Complete sequence of the 45-kb mouse ribosomal DNA repeat: analysis of the intergenic spacer. Genomics 82, 637-643.

Grummt I. (2007). Different epigenetic layers engage in complex crosstalk to define the epigenetic state of mammalian rRNA genes. Hum Mol Gene 16, R21-R27.

Guo J, Li T, Schipper J, Nilson KA, Fordjour FK, Cooper JJ, Gordan R, Price DH. (2014). Sequence specificity incompletely defines the genome-wide occupancy of Myc. Genome Biol 15, 482.

Haltiner MM, Smale ST \& Tjian R. (1986). Two distinct promoter elements in the human rRNA gene identified by linker scanning mutagenesis. Mol Cell Biol 6, 227-235.

Hammarstrom D, Ofsteng S, Koll L, Hanestadhaugen M, Hollan I, Apro W, Whist JE, Blomstrand E, Ronnestad BR \& Ellefsen S. (2020). Benefits of higher resistance-training volume are related to ribosome biogenesis. J Physiol 598, 543-565.

Hansson B, Olsen LA, Nicoll JX, von Walden F, Melin M, Strömberg A, Rullman E, Gustafsson T, Fry AC, Fernandez-Gonzalo R, Lundberg TR. (2019) Skeletal muscle signaling responses to resistance exercise of the elbow extensors are not compromised by a preceding bout of aerobic exercise. Am J Physiol Reg Comp Integr Physiol. 317, R83-92.

Ivey FM, Roth SM, Ferrell RE, Tracy BL, Lemmer JT, Hurlbut DE, Martel GF, Siegel EL, Fozard JL, Jeffrey Metter E, Fleg JL \& Hurley BF. (2000). Effects of age, gender, and myostatin genotype on the hypertrophic response to heavy resistance strength training. $J$ Gerentol Ser A 55, M641-648.

Iwata M, Englund DA, Wen Y, Dungan CM, Murach KA, Vechetti IJ, Mobley CB, Peterson CA \& McCarthy JJ. (2018). A novel tetracycline-responsive transgenic mouse strain for skeletal muscle-specific gene expression. Skelet Muscle 8, 33.

Jozsi AC, Dupont-Versteegden EE, Taylor-Jones JM, Evans WJ, Trappe TA, Campbell WW \& Peterson CA. (2000). Aged human muscle demonstrates an altered gene expression profile consistent with an impaired response to exercise. Mech Age Dev 120, 45-56.

Kim HG, Guo B \& Nader GA. (2019). Regulation of ribosome biogenesis during skeletal muscle hypertrophy. Exerc Sport Sci Rev 47, 91-97. 
865

866

867

868

869

870

871

872

873

874

875

876

877

878

879

880

881

882

883

884

885

886

887

888

889

890

891

892

893

894

895

896

897

898

899

900

901

902

903

904

905

906

907

908

909

910

911

912

913

914

915

Kim S, Li Q, Dang CV \& Lee LA. (2000). Induction of ribosomal genes and hepatocyte hypertrophy by adenovirus-mediated expression of c-Myc in vivo. Proc Nat Acad Sci 97, 11198-11202.

Kirby TJ, Patel RM, McClintock TS, Dupont-Versteegden EE, Peterson CA \& McCarthy JJ. (2016a). Myonuclear transcription is responsive to mechanical load and DNA content but uncoupled from cell size during hypertrophy. Mol Biol Cell 27, 788-798.

Langmead B \& Salzberg SL. (2012). Fast gapped-read alignment with Bowtie 2. Nat Meth 9, 357.

Langmead B, Wilks C, Antonescu V \& Charles R. (2019). Scaling read aligners to hundreds of threads on general-purpose processors. Bioinformatics 35, 421-432.

Lavin KM, Roberts BM, Fry CS, Moro T, Rasmussen BB \& Bamman MM. (2019). The importance of resistance exercise training to combat neuromuscular aging. Physiology 34, 112-122.

Leary DJ \& Huang S. (2001). Regulation of ribosome biogenesis within the nucleolus. FEBS Lett 509, 145-150.

Li X, Wang SJ, Tan SC, Chew PL, Liu L, Wang L, Wen L \& Ma L. (2014). The A55T and K153R polymorphisms of MSTN gene are associated with the strength training-induced muscle hypertrophy among Han Chinese men. J Sports Sci 32, 883-891.

Louis E, Raue U, Yang Y, Jemiolo B, Trappe S. (2007). Time course of proteolytic, cytokine, and myostatin gene expression after acute exercise in human skeletal muscle. J Appl Physiol. $103,1744-51$.

Maasar MF, Turner DC, Gorski PP, Seaborne RA, Strauss JA, Shepherd SO, Cocks M, Pillon NJ, Zierath JR, Hulton AT, Drust B, Sharples AP. (2020). The methylome and comparative transcriptome after high intensity sprint exercise in human skeletal muscle. bioRxiv, https://doi.org/10.1101/2020.09.11.292805.

Malinovskaya EM, Ershova ES, Golimbet VE, Porokhovnik LN, Lyapunova NA, Kutsev SI, Veiko NN \& Kostyuk SV. (2018). Copy number of human ribosomal genes with aging: unchanged mean, but narrowed range and decreased variance in elderly group. Front Gene 9, 306.

Mayer C, Schmitz K-M, Li J, Grummt I \& Santoro R. (2006). Intergenic transcripts regulate the epigenetic state of rRNA genes. Mol Cell 22, 351-361.

McCarthy JJ \& Murach KA. (2019). Anabolic and catabolic signaling pathways that regulate skeletal muscle mass. In Nutrition and Enhanced Sports Performance, pp. 275-290. Elsevier.

Moberg M, Lindholm ME, Reitzner SM, Ekblom B, Sundberg C-J \& Psilander N. (2020). Exercise induces different molecular responses in trained and untrained human muscle. Med Sci Sport Exerc 52, 1679-1690.

Moss T. At the crossroads of growth control; making ribosomal RNA. (2004). Curr Op Gene Dev. 14, 210-7. 
916

917

918

919

920

921

922

923

924

925

926

927

928

929

930

931

932

933

934

935

936

937

938

939

940

941

942

943

944

945

946

947

948

949

950

951

952

953

954

955

956

957

958

959

960

961

962

963

964

965

966

Mougey EB, Pape LK \& Sollner-Webb B. (1996). Virtually the entire Xenopus laevis rDNA multikilobase intergenic spacer serves to stimulate polymerase I transcription. J Biol Chem 271, 27138-27145.

Murach KA \& Bagley JR. (2016). Skeletal muscle hypertrophy with concurrent exercise training: Contrary evidence for an interference effect. Sport Med 46, 1029-1039.

Murach KA, Dungan CM, Dupont-Versteegden EE, McCarthy JJ \& Peterson CA. (2019). "Muscle memory" not mediated by myonuclear number?: Secondary analysis of human detraining data. J Appl Physiol 127.

Murach KA, Mobley CB, Zdunek CJ, Frick KK, Jones SR, McCarthy JJ, Peterson CA \& Dungan CM. (2020). Muscle memory: myonuclear accretion, maintenance, morphology, and miRNA levels with training and detraining in adult mice. J Cachex Sarc Muscle doi: https://doi.org/10.1002/jcsm.12617.

Murayama A, Ohmori K, Fujimura A, Minami H, Yasuzawa-Tanaka K, Kuroda T, Oie S, Daitoku H, Okuwaki M \& Nagata K. (2008). Epigenetic control of rDNA loci in response to intracellular energy status. Cell 133, 627-639.

Nakada S, Ogasawara R, Kawada S, Maekawa T \& Ishii N. (2016). Correlation between Ribosome Biogenesis and the Magnitude of Hypertrophy in Overloaded Skeletal Muscle. PloS One 11, e0147284-e0147284.

Nemeth A, Guibert S, Tiwari VK, Ohlsson R \& Längst G. (2008). Epigenetic regulation of TTF-Imediated promoter-terminator interactions of rRNA genes. EMBO J 27, 1255-1265.

Nie Z, Guo C, Das SK, Chow CC, Batchelor E, Jnr SSS \& Levens D. (2020). Dissecting transcriptional amplification by MYC. eLife $\mathbf{9}$, e52483.

Nie Z, Hu G, Wei G, Cui K, Yamane A, Resch W, Wang R, Green DR, Tessarollo L \& Casellas R. (2012). c-Myc is a universal amplifier of expressed genes in lymphocytes and embryonic stem cells. Cell 151, 68-79.

Ogasawara R, Fujita S, Hornberger TA, Kitaoka Y, Makanae Y, Nakazato K, Naokata I. The role of mTOR signalling in the regulation of skeletal muscle mass in a rodent model of resistance exercise. (2016). Sci Rep. 6, 31142.

Park Y, Figueroa ME, Rozek LS \& Sartor MA. (2014). MethylSig: a whole genome DNA methylation analysis pipeline. Bioinformatics 30, 2414-2422.

Parks MM, Kurylo CM, Dass RA, Bojmar L, Lyden D, Vincent CT \& Blanchard SC. (2018). Variant ribosomal RNA alleles are conserved and exhibit tissue-specific expression. Sci $A d v$ 4, eaao0665.

Perini G, Diolaiti D, Porro A \& Della Valle G. (2005). In vivo transcriptional regulation of N-Myc target genes is controlled by E-box methylation. Proc Nat Acad Sci 102, 12117-12122.

Pietrzak M, Rempala G, Nelson PT, Zheng J-J \& Hetman M. (2011). Epigenetic silencing of nucleolar rRNA genes in Alzheimer's disease. PloS One 6, e22585. 
1002

1003

1004

1005

1006

1007

1008

1009

1010

1011

1012

1013

1014

1015

1016

1017

Pilegaard H, Ordway GA, Saltin B \& Neufer PD. (2000). Transcriptional regulation of gene expression in human skeletal muscle during recovery from exercise. Am J Physiol Endo Metab 279, E806-E814.

Pillon NJ, Gabriel BM, Dollet L, Smith JAB, Puig LS, Botella J, Bishop DJ, Krook A, Zierath JR. (2020). Transcriptional profoiling of skeletal muscle adaptations to exericise and inactivity. Nat Comm 11, 1-15.

Prendergast GC, Lawe D \& Ziff EB. (1991). Association of Myn, the murine homolog of max, with c-Myc stimulates methylation-sensitive DNA binding and ras cotransformation. Cell 65, 395-407.

Riechman SE, Balasekaran G, Roth SM \& Ferrell RE. (2004). Association of interleukin-15 protein and interleukin-15 receptor genetic variation with resistance exercise training responses. J Appl Physiol 97, 2214-2219.

Santoro R, Schmitz KM, Sandoval J \& Grummt I. (2010). Intergenic transcripts originating from a subclass of ribosomal DNA repeats silence ribosomal RNA genes in trans. EMBO Rep $11,52-58$.

Seaborne RA, Strauss J, Cocks M, Shepherd S, O'Brien TD, van Someren KA, Bell PG, Murgatroyd C, Morton JP, Stewart CE \& Sharples AP. (2018). Human skeletal muscle possesses an epigenetic memory of hypertrophy. Sci Rep 8, 1898.

Seeman E, Hopper JL, Young NR, Formica C, Goss P \& Tsalamandris C. (1996). Do genetic factors explain associations between muscle strength, lean mass, and bone density? A twin study. Am J Physiol 270, E320-327.

Sharples AP \& Seaborne RA. (2019). Exercise and DNA methylation in skeletal muscle. In Sports, exercise, and nutritional genomics, pp. 211-229. Elsevier.

Sharples AP, Stewart CE \& Seaborne RA. (2016). Does skeletal muscle have an 'epi'-memory? The role of epigenetics in nutritional programming, metabolic disease, aging and exercise. Aging Cell 15, 603-616.

Shiue C-N, Nematollahi-Mahani A \& Wright AP. (2014). Myc-induced anchorage of the rDNA IGS region to nucleolar matrix modulates growth-stimulated changes in higher-order rDNA architecture. Nuc Acid Res 42, 5505-5517.

Snijders T, Aussieker T, Holwerda A, Parise G, van Loon L \& Verdijk LB. (2020). The concept of skeletal muscle memory: evidence from animal and human studies. Acta Physiol e13465.

Sparks LM. (2017). Exercise training response heterogeneity: physiological and molecular insights. Diabetologia 60, 2329-2336.

Stancheva I, Lucchini R, Koller T \& Sogo JM. (1997). Chromatin structure and methylation of rat rRNA genes studied by formaldehyde fixation and psoralen cross-linking. Nuc Acid Res 25, 1727-1735.

Stec MJ, Kelly NA, Many GM, Windham ST, Tuggle SC \& Bamman MM. (2016). Ribosome biogenesis may augment resistance training-induced myofiber hypertrophy and is required for myotube growth in vitro. Am J Physiol Endo Metab 8, E652-E661. 
1018

1019

1020

1021

1022

1023

1024

1025

1026

1027

1028

1029

1030

1031

1032

1033

1034

1035

1036

1037

1038

1039

1040

1041

1042

1043

1044

1045

1046

1047

1048

1049

1050

1051

1052

1053

1054

1055

1056

1057

1058

1059

1060

1061

1062

1063

1064

1065

1066

1067

1068
Stec MJ, Mayhew DL \& Bamman MM. (2015). The effects of age and resistance loading on skeletal muscle ribosome biogenesis. J Appl Physiol 119, 851-857.

Turner DC, Seaborne RA \& Sharples AP. (2019). Comparative transcriptome and methylome analysis in human skeletal muscle anabolism, hypertrophy and epigenetic memory. Sci Rep 9, 1-12.

Uemura M, Zheng Q, Koh CM, Nelson WG, Yegnasubramanian S \& De Marzo AM. (2012). Overexpression of ribosomal RNA in prostate cancer is common but not linked to rDNA promoter hypomethylation. Oncogene 31, 1254-1263.

Vacík T, Kereïche S, Raška I, Cmarko D \& Smirnov E. (2019). Life time of some RNA products of rDNA intergenic spacer in HeLa cells. Histochem Cell Biol 152, 271-280.

Van Riggelen J, Yetil A \& Felsher DW. (2010). MYC as a regulator of ribosome biogenesis and protein synthesis. Nat Rev Cancer 10, 301.

Veal E \& Jackson M. (1998). C-myc is expressed in mouse skeletal muscle nuclei during postnatal maturation. Int J Biochem Cell Biol 30, 811-821.

Vissing K, McGee SL, Farup J, Kjølhede T, Vendelbo MH, Jessen N. (2013). Differentiated mTOR but not AMPK signaling after strength vs endurance exercise in trainingaccustomed individuals. Scand J Med Sci Sport 23, 355-66.

von Walden F, Rea M, Mobley CB, Fondufe-Mittendorf Y, McCarthy JJ, Peterson CA \& Murach KA. (2020a). The myonuclear DNA methylome in response to an acute hypertrophic stimulus. Epigenetics 15, 1151-1162.

von Walden F. (2019). Ribosome biogenesis in skeletal muscle: coordination of transcription and translation. J Appl Physiol 127, 591-598.

von Walden F, Casagrande V, Östlund Farrants A-K \& Nader GA. (2012). Mechanical loading induces the expression of a Pol I regulon at the onset of skeletal muscle hypertrophy. Am J Physiol Cell Physiol 302, C1523-C1530.

von Walden F, Fernandez-Gonzalo R, Pingel J, McCarthy J, Stål P \& Pontén E. (2020b). Epigenetic marks at the ribosomal DNA promoter in skeletal muscle are negatively associated with degree of impairment in cerebral palsy. Front Ped 8, 236.

von Walden F, Liu C, Aurigemma N \& Nader GA. (2016). mTOR signaling regulates myotube hypertrophy by modulating protein synthesis, rDNA transcription and chromatin remodeling. Am J Physiol Cell Physiol, ajpcell.00144.02016-ajpcell.00144.02016.

Wen Y, Alimov AP \& McCarthy JJ. (2016). Ribosome biogenesis is necessary for skeletal muscle hypertrophy. Exerc Sport Sci Rev 44, 110.

Wen Y, Vechetti Jr IJ, Valentino TR \& McCarthy JJ. (2020). High-yield skeletal muscle protein recovery from TRIzol after RNA and DNA extraction. BioTechniques 69, 264-269. 
1069

1070

1071

1072

1073

1074

1075

1076

1077

1078

1079

1080

1081

1082

1083

1084

1085

1086

1087

1088

1089

1090

1091

1092

1093

1094

1095

1096

1097

1098

1099

1100

1101

1102

1103

1104

1105

1106

1107

1108

1109
West DW, Baehr LM, Marcotte GR, Chason CM, Tolento L, Gomes AV, Bodine SC \& Baar K. (2016). Acute resistance exercise activates rapamycin-sensitive and-insensitive mechanisms that control translational activity and capacity in skeletal muscle. J Physiol 594, 453-468.

Xiao G, Mao S, Baumgarten G, Serrano J, Jordan MC, Roos KP, Fishbein MC \& MacLellan WR. (2001). Inducible activation of c-Myc in adult myocardium in vivo provokes cardiac myocyte hypertrophy and reactivation of DNA synthesis. Circ Res 89, 1122-1129.

Zentner GE, Balow SA \& Scacheri PC. (2014). Genomic characterization of the mouse ribosomal DNA locus. G3 4, 243-254.

Zentner GE, Saiakhova A, Manaenkov P, Adams MD \& Scacheri PC. (2011a). Integrative genomic analysis of human ribosomal DNA. Nuc Acid Res 39, 4949-4960.

Zentner GE, Tesar PJ \& Scacheri PC. (2011b). Epigenetic signatures distinguish multiple classes of enhancers with distinct cellular functions. Genome Res 21, 1273-1283.

Zhong W, Mao S, Tobis S, Angelis E, Jordan MC, Roos KP, Fishbein MC, de Alborán IM \& MacLellan WR. (2006). Hypertrophic growth in cardiac myocytes is mediated by Myc through a Cyclin D2-dependent pathway. EMBO J 25, 3869-3879. 


\section{Competing Interests}

1111 None of the authors have any conflict of interest to disclose.

\section{Author Contributions}

1114 VCF conducted experiments and data analysis, generated figures, and drafted the manuscript

1115 YW conducted experiments and data analysis, generated figures, and assisted with manuscript 1116 preparation

1117 BA coordinated the study, assisted with data collection, and provided resources

1118 RF-G assisted with data collection and provided resources

$1119 \mathrm{JN}$ assisted with data collection and provided resources

1120 IV conducted experiments and data analysis

1121 TV conducted experiments

1122 CBM conducted experiments

1123 GEZ assisted with manuscript preparation, figure preparation, and data analysis

1124 CAP provided resources

1125 JJM provided resources

1126 KAM coordinated the study, conducted experiments and data analysis, generated figures and the

1127 graphical abstract, provided resources, and drafted the manuscript

1128 FvW coordinated the study, conducted experiments and data analysis, generated figures, provided

1129 resources, and drafted the manuscript

1130 All authors read, edited, and approved of the final manuscript

\section{Funding}

1133 The study was funded by Futurum - the Academy for Health and Care, Region Jönköping

1134 County, Sweden to BA and the Swedish Kidney Foundation and Swedish research council for

1135 Sports to FvW, and a National Institutes of Health grant (NIH K99 AG063994) to KAM.

\section{Acknowledgements}

1137 The authors would like to thank the subjects for their extraordinary effort and participation. We 1138 further greatly acknowledge the help from staff at Höglandssjukhuset District Hospital in Eksjö 1139 where the human study took place, especially Annica Eriksson, Lena Norrbrand, Björn Otto, and 1140 the personnel at the Orthopaedic Department. We wish to thank Jennifer Strange of the University 1141 of Kentucky Flow Cytometry Core, as well as Dr. Keith Booher at Zymo Research. The graphical 1142 abstract was generated using BioRender. 